\title{
Generalized trapezium-type inequalities in the settings of fractal sets for functions having generalized convexity property
}

\author{
Zareen A. Khan', Saima Rashid², Rehana Ashraf' ${ }^{3}$, Dumitru Baleanu ${ }^{4}$ and Yu-Ming Chu ${ }^{5,6 *}$
}

\author{
"Correspondence: \\ chuyuming2005@126.com \\ ${ }^{5}$ Department of Mathematics, \\ Huzhou University, Huzhou, China \\ ${ }^{6}$ Hunan Provincial Key Laboratory of \\ Mathematical Modeling and \\ Analysis in Engineering, Changsha \\ University of Science \& Technology, \\ Changsha, China \\ Full list of author information is \\ available at the end of the article
}

\begin{abstract}
In the paper, we extend some previous results dealing with the Hermite-Hadamard inequalities with fractal sets and several auxiliary results that vary with local fractional derivatives introduced in the recent literature. We provide new generalizations for the third-order differentiability by employing the local fractional technique for functions whose local fractional derivatives in the absolute values are generalized convex and obtain several bounds and new results applicable to convex functions by using the generalized Hölder and power-mean inequalities.

As an application, numerous novel cases can be obtained from our outcomes. To ensure the feasibility of the proposed method, we present two examples to verify the method. It should be pointed out that the investigation of our findings in fractal analysis and inequality theory is vital to our perception of the real world since they are more realistic models of natural and man-made phenomena.
\end{abstract}

MSC: 26D15; 26D10; 90C23

Keywords: Generalized convex functions; Hermite-Hadamard inequalty; Čebyšev inequality; Generalized Hölder inequality; Power-mean inequality; Fractal set

\section{Introduction}

Fractals are mathematical developments that present self-similarity over a scope of scales and noninteger (fractal) measurements. Attributable to these properties, Yang [1] contemplated a new investigation to tackle some nondifferentiable problems that can be utilized to productively assess the geometrical multifaceted nature, and the anomaly of shapes and examples seen in human disease through the utilization of customary Euclidean geometry in such figurings is all the more challenging $[2,3]$. The use of fractal analysis in image processing, machine learning, cryptography, electrochemical processes, physics, diagnostic imagining, neuroscience, image analysis, acoustic, physiology, and Riemann zeta zeros has shown considerable guarantee for estimating forms that have changed as ordinarypartial differential equations [4-8]. Regardless of the benefits of fractal mathematics and various examinations exhibiting its pertinence to porous media, aquifer, turbulence, and more other media commonly displaying fractal properties, numerous specialists and researchers stay uninformed of its latent capacity involving local fractional articulations.

(c) The Author(s) 2020. This article is licensed under a Creative Commons Attribution 4.0 International License, which permits use sharing, adaptation, distribution and reproduction in any medium or format, as long as you give appropriate credit to the original author(s) and the source, provide a link to the Creative Commons licence, and indicate if changes were made. The images or other third party material in this article are included in the article's Creative Commons licence, unless indicated otherwise in a credit line to the material. If material is not included in the article's Creative Commons licence and your intended use is not permitted by statutory regulation or exceeds the permitted use, you will need to obtain permission directly from the copyright holder. To view a copy of this licence, visit http://creativecommons.org/licenses/by/4.0/. 
Moreover, local fractional derivatives and integrals are viably applied numerous conditions, for instance, the Fokker-Planck equation, the diffusion and relaxation equation in fractal space, the fractal heat conduction equation, and the local fractional diffusion equation [9]. Moreover, fractional calculus (integrals and derivatives) has gained significant interest throughout the past three decades due chiefly to its incontestable utilities in numerous areas of science and technology $[10,11]$. The fractional operator does indeed provide many potentially helpful tools for numerous problems involving special functions of mathematical science and their extensions and generalizations in one and several variables. Fractional integrals are utilized for depiction of various hereditary and memory effects of different processes and constituents in physical processes like seepage flow in fluid dynamic traffic model and nonlinear oscillations of earthquake [12, 13].

Fractional integrals have been analyzed for integral inequalities and solution of fractional differential and difference equations. The Hermite-Hadamard inequality [14] is one of such type inequalities, extensively used in the literature and providing a necessary and sufficient condition for a function to be convex.

Now we recall the Hermite-Hadamard inequality. Let $\mathcal{G}: \Omega \subseteq \mathbb{R} \rightarrow \mathbb{R}$ be a convex function. Then we have the double inequality

$$
\mathcal{G}\left(\frac{\eta_{1}+\eta_{2}}{2}\right) \leq \frac{1}{\eta_{2}-\eta_{1}} \int_{\eta_{1}}^{\eta_{2}} \mathcal{G}(\delta) d \delta \leq \frac{\mathcal{G}\left(\eta_{1}\right)+\mathcal{G}\left(\eta_{2}\right)}{2}
$$

for all $\eta_{1}, \eta_{2} \in \Omega$ with $\eta_{1} \neq \eta_{2}$.

Recently, generalizations, extensions, improvements, and variants of the HermiteHadamard inequality have attracted the attention of many researchers due to its wide applications in pure and applied mathematics. The main purpose of the paper is presenting the fundamental basis of fractals and illustrating analysis of fractal sets and related estimations, in particular, establishing the integral inequalities for the functions whose local fractional differentiation in the absolute values are generalized convex.

Local fractional inequalities and their fertile applications in pure and applied mathematics have attracted the attention of many researchers $[15,16]$. For example, Mo et al. [17] derived the generalized Hermite-Hadamard inequality for generalized convex functions. Chen et al. [18] explored it extensively by using the Hölder inequality and some other related variants in a fractal domain. The concept of a generalized harmonically convex function was introduced by Sun [19].

In this paper, we investigate new concepts of differentiation and integration taking into account the fractal sets and generalized convex functions. We present other important auxiliary results, handled by this new approach for higher-order local differentiability, which enable us to give certain estimates of the difference between the left and middle parts of the Hermite-Hadamard inequality. On fractal sets, we carry out two examples illustrating the applicability of the proposed methodology. As an application, we derived some novel cases in local fractional trapezoid form. Generalized new special cases show an impressive performance of the local fractional integration. Some special cases are correlated with existing results on classical convexity. 


\section{Preliminaries}

In this section, we present a different concept of differentiation, which consolidates the ideas of fractional differentiation and fractal derivative. We denote the sets of natural numbers, positive integers, rational numbers, and real numbers by $\mathbb{N}, \mathbb{Z}, \mathbb{Q}$, and $\mathbb{R}$, respectively.

Whenever we consider the $\check{\alpha}$-type set $\mathbb{R}^{\check{\alpha}}$ of real line numbers, we implicitly suppose that $0<\check{\alpha} \leq 1$. Two binary operations, the addition "+" and the multiplication "." (which is conventionally omitted), on the $\check{\alpha}$-type set $\mathbb{R}^{\check{\alpha}}$ of real line numbers are characterized as follows. Let $\zeta_{1}^{\check{\alpha}}, \zeta_{2}^{\check{\alpha}} \in \mathbb{R}^{\check{\alpha}}$. Then the addition "+" and multiplication "." are defined by $\zeta_{1}^{\check{\alpha}}+\zeta_{2}^{\check{\alpha}}=\left(\zeta_{1}+\zeta_{2}\right)^{\check{\alpha}}$ and $\zeta_{1}^{\check{\alpha}} \cdot \zeta_{2}^{\check{\alpha}}=\left(\zeta_{1} \cdot \zeta_{2}\right)^{\check{\alpha}}$, respectively.

We have the following statements:

- If $\left(\mathbb{R}^{\check{\alpha}},+\right)$ is an Abelian group and $\zeta_{1}^{\check{\alpha}}, \zeta_{2}^{\check{\alpha}}, \zeta_{3}^{\check{\alpha}} \in \mathbb{R}^{\check{\alpha}}$. then

(i) $\zeta_{1}^{\check{\alpha}}+\zeta_{2}^{\check{\alpha}} \in \mathbb{R}^{\check{\alpha}}$;

(ii) $\zeta_{1}^{\check{\alpha}}+\zeta_{2}^{\check{\alpha}}=\zeta_{2}^{\check{\alpha}}+\zeta_{1}^{\check{\alpha}}$;

(iii) $\zeta_{1}^{\check{\alpha}}+\left(\zeta_{2}^{\check{\alpha}}+\zeta_{3}^{\check{\alpha}}\right)=\left(\zeta_{1}^{\check{\alpha}}+\zeta_{2}^{\check{\alpha}}\right)+\zeta_{3}^{\check{\alpha}}$;

(iv) $0^{\check{\alpha}}+\zeta_{1}^{\check{\alpha}}=\zeta_{1}^{\check{\alpha}}+0^{\check{\alpha}}=\zeta_{1}^{\check{\alpha}}$ (where $0^{\check{\alpha}}$ is the additive identity of $\left.\left(\mathbb{R}^{\check{\alpha}},+\right)\right)$;

(v) $\zeta_{1}^{\check{\alpha}}+\left(-\zeta_{1}^{\check{\alpha}}\right)=\left(-\zeta_{1}^{\check{\alpha}}\right)+\zeta_{1}^{\check{\alpha}}=0^{\check{\alpha}}$ (where $-\zeta^{\check{\alpha}}$ is the inverse element of $\left.\zeta^{\check{\alpha}}\right)$;

• If $\left(\mathbb{R}^{\check{\alpha}} \backslash\left\{0^{\check{\alpha}}\right\}, \cdot\right)$ is an Abelian group and $\zeta_{1}^{\check{\alpha}}, \zeta_{2}^{\check{\alpha}}, \zeta_{3}^{\check{\alpha}} \in \mathbb{R}^{\check{\alpha}}$, then

(vi) $\zeta_{1}^{\check{\alpha}} \cdot \zeta_{2}^{\check{\alpha}} \in \mathbb{R}^{\check{\alpha}}$;

(vii) $\zeta_{1}^{\check{\alpha}} \cdot \zeta_{2}^{\check{\alpha}}=\zeta_{2}^{\check{\alpha}} \cdot \zeta_{1}^{\check{\alpha}}$;

$\left(\right.$ viii) $\zeta_{1}^{\check{\alpha}} \cdot\left(\zeta_{2}^{\check{\alpha}} \cdot \zeta_{3}^{\check{\alpha}}\right)=\left(\zeta_{1}^{\check{\alpha}} \cdot \zeta_{2}^{\check{\alpha}}\right) \cdot \zeta_{3}^{\check{\alpha}}$

(ix) $1^{\check{\alpha}} \cdot \zeta_{1}^{\check{\alpha}}=\zeta_{1}^{\check{\alpha}} \cdot 1^{\check{\alpha}}=\zeta_{1}^{\check{\alpha}}\left(\right.$ where $1^{\check{\alpha}}$ is the multiplicative identity of $\left.\left(\mathbb{R}^{\check{\alpha}}, \cdot\right)\right)$;

$(x) \zeta_{1}^{\check{\alpha}} \cdot\left(\frac{1}{\zeta_{1}}\right)^{\check{\alpha}}=\left(\frac{1}{\zeta_{1}}\right)^{\check{\alpha}} \cdot \zeta_{1}^{\check{\alpha}}=1^{\check{\alpha}}$ (where $\left(\frac{1}{\zeta}\right)^{\check{\alpha}}$ is the inverse element of $\left.\zeta^{\check{\alpha}}\right)$.

•. The distributive law: $\zeta_{1}^{\check{\alpha}} \cdot\left(\zeta_{2}^{\check{\alpha}}+\zeta_{3}^{\check{\alpha}}\right)=\zeta_{1}^{\check{\alpha}} \cdot \zeta_{2}^{\check{\alpha}}+\zeta_{1}^{\check{\alpha}} \cdot \zeta_{3}^{\check{\alpha}}$ for all $\zeta_{1}^{\check{\alpha}}, \zeta_{2}^{\check{\alpha}} \in \mathbb{R}^{\check{\alpha}}$.

Proposition 2.1 We have:

(i) $\left(\mathbb{R}^{\check{\alpha}},+, \cdot\right)$ is a field;

(ii) Both the additive identity $0^{\check{\alpha}}$ and the multiplication identity $1^{\check{\alpha}}$ are unique;

(iii) Both the additive inverse element and the multiplicative inverse element are unique;

(iv) For each $\zeta_{1}^{\check{\alpha}} \in \mathbb{R}^{\check{\alpha}} \backslash\left\{0^{\check{\alpha}}\right\}$, its inverse element $\left(\frac{1}{\zeta_{1}}\right)^{\check{\alpha}}$ can be written as $\frac{1^{\check{\alpha}}}{\zeta_{1}^{\alpha}}$ or as $\frac{1}{\zeta_{1}^{\check{\alpha}}}$; for each $\zeta_{1}^{\check{\alpha}} \in \mathbb{R}^{\check{\alpha}}$, its inverse element $\left(-\zeta_{1}\right)^{\check{\alpha}}$ can be written as $-\zeta_{1}^{\check{\alpha}}$;

(v) The order " $<$ " in $\left(\mathbb{R}^{\check{\alpha}},+\right)$ is defined as follows: $\zeta_{1}^{\check{\alpha}}<\zeta_{2}^{\check{\alpha}} \in \mathbb{R}^{\check{\alpha}}$ if and only if $\zeta_{1}<\zeta_{2} \in \mathbb{R}$. In particular, $\left(\mathbb{R}^{\check{\alpha}},+, \cdot,<\right)$ is an ordered field like $(\mathbb{R},+, \cdot,<)$.

Now let us demonstrate the idea of the local fractional continuity.

Definition 2.2 Let $\mathcal{G}: \mathbb{R} \rightarrow \mathbb{R}^{\check{\alpha}}$ be a nondifferentiable mapping. Then $\rho \rightarrow \mathcal{G}(\epsilon)$ is said to be locally fractional continuous at $\epsilon_{\mathrm{o}}$ if for any $\epsilon>0$, there exists $\kappa>0$ such that

$$
\left|\mathcal{G}(\epsilon)-\mathcal{G}\left(\epsilon_{\circ}\right)\right|<\epsilon^{\check{\alpha}}
$$

whenever $\left|\epsilon-\epsilon_{\circ}\right|<\kappa$. If $\mathcal{G}(\epsilon)$ is locally continuous on $\left(\eta_{1}, \eta_{2}\right)$, then we write $\mathcal{G}(\epsilon) \epsilon$ $\mathbb{C}_{\check{\alpha}}\left(\eta_{1}, \eta_{2}\right)$.

Definition 2.3 The local fractional derivative of $\mathcal{G}(\epsilon)$ of order $\check{\alpha}$ at $\epsilon=\epsilon_{\circ}$ is defined by

$$
\mathcal{G}^{(\check{\alpha})}\left(\epsilon_{\circ}\right)=\epsilon_{\circ} \mathcal{D}_{\epsilon}^{\check{\alpha}} \mathcal{G}(\epsilon)=\left.\frac{d^{\check{\alpha}} \mathcal{G}(\epsilon)}{d \epsilon^{\check{\alpha}}}\right|_{\epsilon=\epsilon_{\circ}}=\lim _{\epsilon \rightarrow \epsilon_{\circ}} \frac{\Delta^{\check{\alpha}}\left(\mathcal{G}(\epsilon)-\mathcal{G}\left(\epsilon_{\circ}\right)\right)}{\left(\epsilon-\epsilon_{\circ}\right)^{\check{\alpha}}}
$$


where $\Delta^{\check{\alpha}}\left(\mathcal{G}(\epsilon)-\mathcal{G}\left(\epsilon_{\circ}\right)\right)=\Gamma(\check{\alpha}+1)\left(\mathcal{G}(\epsilon)-\mathcal{G}\left(\epsilon_{\circ}\right)\right)$. We also write $\mathcal{G}^{(\check{\alpha})}(\epsilon)=\mathcal{D}_{\epsilon}^{\check{\alpha}} \mathcal{G}(\epsilon)$. If there $(k+1)$ times

exists $\mathcal{G}^{(k+1) \check{\alpha}}(\epsilon)=\overbrace{\mathcal{D}_{\epsilon}^{\check{\alpha}} \cdots \mathcal{D}_{\epsilon}^{\check{\alpha}}}^{\mathcal{G}}(\epsilon)$ for all $\epsilon \in \Omega \subseteq \mathbb{R}$, then we write $\mathcal{G} \in \mathcal{D}_{(k+1) \check{\alpha}}(\Omega)$, where $k=0,1,2, \ldots$.

Definition 2.4 Let $\mathcal{G}(\epsilon) \in \mathbb{C}_{\check{\alpha}}\left[\eta_{1}, \eta_{2}\right]$, and let $\Delta=\left\{\varrho_{0}, \varrho_{1}, \ldots, \varrho_{N}\right\}(N \in \mathbb{N})$ be a partition of $\left[\eta_{1}, \eta_{2}\right]$ such that $\eta_{1}=\varrho_{0}<\varrho_{1}<\cdots<\varrho_{N}=\eta_{2}$. Then the local fractional integral of $\mathcal{G}$ on $\left[\eta_{1}, \eta_{2}\right]$ of order $\check{\alpha}$ is defined by

$$
\eta_{1} \mathcal{I}_{\eta_{2}}^{(\check{\alpha})} \mathcal{G}(\epsilon)=\frac{1}{\Gamma(1+\check{\alpha})} \int_{\eta_{1}}^{\eta_{2}} \mathcal{G}(\varrho)(d \varrho)^{\check{\alpha}}=\frac{1}{\Gamma(1+\check{\alpha})} \lim _{\delta} \sum_{j=0}^{N-1} \mathcal{G}\left(\varrho_{j}\right)\left(\Delta \varrho_{j}\right),
$$

where $\delta \varrho=\max \left\{\Delta \varrho_{1}, \Delta \varrho_{2}, \ldots, \Delta \varrho_{N-1}\right\}$ and $\Delta \varrho_{j}=\varrho_{j+1}-\varrho_{j}(j=0, \ldots, N-1)$. It follows that ${ }_{\eta_{1}} \mathcal{I}_{\eta_{2}}^{(\breve{\alpha})} \mathcal{G}(\epsilon)=0$ if $\eta_{1}=\eta_{2}$ and ${ }_{\eta_{1}} \mathcal{I}_{\eta_{2}}^{(\check{\alpha})} \mathcal{G}(\epsilon)=-{ }_{\eta_{2}} \mathcal{I}_{\eta_{1}}^{(\breve{\alpha})} \mathcal{G}(\epsilon)$ if $\eta_{1}<\eta_{2}$. If for all $\epsilon \in\left[\eta_{1}, \eta_{2}\right]$, there exists ${ }_{\eta_{1}} \mathcal{I}_{\eta_{2}}^{(\check{\alpha})} \mathcal{G}(\epsilon)$, then we write $\mathcal{G}(\epsilon) \in \mathcal{I}_{\epsilon}^{\check{\alpha}}\left[\eta_{1}, \eta_{2}\right]$.

Lemma 2.5 (See [1]) We have:

(1) If $\mathcal{G}(u)=\mathcal{G}^{(\breve{\alpha})}(u) \in \mathbb{C}_{\check{\alpha}}\left[\eta_{1}, \eta_{2}\right]$, then

$$
{ }_{\eta_{1}} \mathcal{I}_{\eta_{2}}^{(\breve{\alpha})} \mathcal{G}(u)=\mathcal{G}\left(\eta_{2}\right)-\mathcal{G}\left(\eta_{1}\right)
$$

(2) If $\mathcal{G}(u), \mathcal{H}(u) \in \mathcal{D}_{\check{\alpha}}\left[\eta_{1}, \eta_{2}\right]$ and $\mathcal{G}^{(\check{\alpha})}(u), \mathcal{H}^{(\check{\alpha})}(u) \in \mathbb{C}_{\check{\alpha}}\left[\eta_{1}, \eta_{2}\right]$, then

$$
{ }_{\eta_{1}} \mathcal{I}_{\eta_{2}}^{(\breve{\alpha})} \mathcal{G}(u) \mathcal{H}^{(\check{\alpha})}(u)=\left.\mathcal{G}(u) \mathcal{H}(u)\right|_{\eta_{1}} ^{\eta_{2}}-{ }_{\eta_{1}} \mathcal{I}_{\eta_{2}}^{(\check{\alpha})} \mathcal{G}^{(\check{\alpha})}(u) \mathcal{H}(u) .
$$

Lemma 2.6 (See [1]) We have the formulas

$$
\begin{aligned}
& \frac{d^{\check{\alpha}} u^{k \check{\alpha}}}{d u^{\check{\alpha}}}=\frac{\Gamma(1+k \check{\alpha})}{\Gamma(1+(k-1) \check{\alpha})} u^{(k-1) \check{\alpha}}, \\
& \frac{1}{\Gamma(1+\check{\alpha})} \int_{\eta_{1}}^{\eta_{2}} u^{k \check{\alpha}}(d u)^{\check{\alpha}}=\frac{\Gamma(1+k \check{\alpha})}{\Gamma(1+(k+1) \check{\alpha})}\left(\eta_{2}^{(k+1) \check{\alpha}}-\eta_{1}^{(k+1) \check{\alpha}}\right) \quad(k>0) .
\end{aligned}
$$

The following analogue of the classical Hölder inequality for a fractal set $\mathbb{R}^{\check{\alpha}}$ was established in [18].

Lemma 2.7 (See [18]) Let $\omega, \rho>1$ with $\omega^{-1}+\rho^{-1}=1$, and let $\mathcal{G}, \mathcal{H} \in \mathbb{C}_{\check{\alpha}}\left[\eta_{1}, \eta_{2}\right]$. Then

$$
\begin{aligned}
& \frac{1}{\Gamma(1+\check{\alpha})} \int_{\eta_{1}}^{\eta_{2}}|\mathcal{G}(u) \mathcal{H}(u)|(d u)^{\check{\alpha}} \\
& \quad \leq\left(\frac{1}{\Gamma(1+\check{\alpha})} \int_{\eta_{1}}^{\eta_{2}}|\mathcal{G}(u)|^{\omega}(d u)^{\check{\alpha}}\right)^{\frac{1}{\omega}}\left(\frac{1}{\Gamma(1+\check{\alpha})} \int_{\eta_{1}}^{\eta_{2}}|\mathcal{H}(u)|^{\rho}(d u)^{\check{\alpha}}\right)^{\frac{1}{\rho}} .
\end{aligned}
$$

Definition 2.8 (See [17]) A function $\mathcal{G}: \Omega \subseteq \mathbb{R} \rightarrow \mathbb{R}^{\check{\alpha}}$ is said to be a generalized convex function on $\Omega$ if

$$
\mathcal{G}\left(\rho \delta_{1}+(1-\rho) \delta_{2}\right) \leq \rho^{\check{\alpha}} \mathcal{G}\left(\delta_{1}\right)+(1-\rho) \mathcal{G}^{\check{\alpha}}\left(\delta_{2}\right)
$$

for all $\delta_{1}, \delta_{2} \in \Omega$ and $\rho \in[0,1]$. 
We provide two examples for generalized convex functions:

(1) $\mathcal{G}(\delta)=\delta^{\check{\alpha} \mu}$ for $\delta \geq 0$ and $\mu>1$.

(2) $\mathcal{G}(\delta)=E_{\check{\alpha}}\left(\delta^{\check{\alpha}}\right)$ for $\delta \in \mathbb{R}$, where $E_{\check{\alpha}}\left(\delta^{\check{\alpha}}\right)=\sum_{i=0}^{\infty} \frac{\delta^{\check{\alpha} i}}{\Gamma(1+i \check{\alpha})}$ is the Mittag-Leffler function.

Recently, the fractal theory has achieved a considerable interest. Mo et al. [17] found the following analogue of the Hermite-Hadamard inequality (1.1) for generalized convex functions:

$$
\mathcal{G}\left(\frac{\eta_{1}+\eta_{2}}{2}\right) \leq \frac{\Gamma(1+\check{\alpha})_{\eta_{1}} \mathcal{I}_{\eta_{2}}^{(\check{\alpha})} \mathcal{G}(u)}{\left(\eta_{2}-\eta_{1}\right)^{\check{\alpha}}} \leq \frac{\mathcal{G}\left(\eta_{1}\right)+\mathcal{G}\left(\eta_{2}\right)}{2^{\check{\alpha}}}
$$

\section{Identity via first-order local differentiable mappings}

To establish our main results, we need the following lemma.

Lemma 3.1 Let $\mathcal{G}: \Omega \rightarrow \mathbb{R}^{\check{\alpha}}(0<\check{\alpha} \leq 1)$ be such that $\mathcal{G} \in \mathcal{D}_{\check{\alpha}}(\Omega)$ and $\mathcal{G}^{(\check{\alpha})} \in \mathcal{C}_{\check{\alpha}}\left[\eta_{1}, \eta_{2}\right]$. Then we have

$$
\begin{aligned}
& \frac{\left(y-\eta_{1}\right)^{\check{\alpha}} \mathcal{G}\left(\eta_{1}\right)+\left(\eta_{2}-y\right)^{\check{\alpha}} \mathcal{G}\left(\eta_{2}\right)}{\left(\eta_{2}-\eta_{1}\right)^{\check{\alpha}}}-\frac{\Gamma(1+\check{\alpha})}{\left(\eta_{2}-\eta_{1}\right)^{\check{\alpha}} \eta_{1}} \mathcal{I}_{\eta_{2}}^{(\check{\alpha})} \mathcal{G}(u) \\
& =\frac{\left(y-\eta_{1}\right)^{2 \check{\alpha}}}{\left(\eta_{2}-\eta_{1}\right)^{\check{\alpha}}} \frac{1}{\Gamma(1+\check{\alpha})} \int_{0}^{1}(\rho-1)^{\check{\alpha}} \mathcal{G}^{(\check{\alpha})}\left(\rho y+(1-\rho) \eta_{1}\right)(d \rho)^{\check{\alpha}} \\
& \quad+\frac{\left(\eta_{2}-y\right)^{2 \check{\alpha}}}{\left(\eta_{2}-\eta_{1}\right)^{\check{\alpha}}} \frac{1}{\Gamma(1+\check{\alpha})} \int_{0}^{1}(1-\rho)^{\check{\alpha}} \mathcal{G}^{(\check{\alpha})}\left(\rho y+(1-\rho) \eta_{2}\right)(d \rho)^{\check{\alpha}},
\end{aligned}
$$

where $\Gamma(x)=\int_{0}^{\infty} t^{x-1} e^{-t} d t$ is the Euler gamma function [20-22].

Proof By using local fractional integration by parts and change of variable we have

$$
\begin{aligned}
& \frac{\left(y-\eta_{1}\right)^{2 \check{\alpha}}}{\left(\eta_{2}-\eta_{1}\right)^{\check{\alpha}}} \frac{1}{\Gamma(1+\check{\alpha})} \int_{0}^{1}(\rho-1)^{\check{\alpha}} \mathcal{G}^{(\check{\alpha})}\left(\rho y+(1-\rho) \eta_{1}\right)(d \rho)^{\check{\alpha}} \\
& =\frac{\left(y-\eta_{1}\right)^{2 \check{\alpha}}}{\left(\eta_{2}-\eta_{1}\right)^{\check{\alpha}}}\left[\left.\frac{(\rho-1)^{\check{\alpha}} \mathcal{G}\left(\rho y+(1-\rho) \eta_{1}\right)}{\left(y-\eta_{1}\right)^{\check{\alpha}}}\right|_{0} ^{1}\right. \\
& \left.\quad-\frac{1}{\left(y-\eta_{1}\right)^{\check{\alpha}}} \frac{\Gamma(1+\check{\alpha})}{\Gamma(1+\check{\alpha})} \int_{0}^{1} \mathcal{G}\left(\rho y+(1-\rho) \eta_{1}\right)(d \rho)^{\check{\alpha}}\right] \\
& =\frac{\left(y-\eta_{1}\right)^{2 \check{\alpha}}}{\left(\eta_{2}-\eta_{1}\right)^{\check{\alpha}}}\left[\frac{\mathcal{G}\left(\eta_{1}\right)}{\left(y-\eta_{1}\right)^{\check{\alpha}}}-\left(\frac{1}{y-\eta_{1}}\right)^{2 \check{\alpha}} \frac{\Gamma(1+\check{\alpha})}{\Gamma(1+\check{\alpha})} \int_{\eta_{1}}^{y} \mathcal{G}(u)(d u)^{\check{\alpha}}\right] \\
& =\frac{\left(y-\eta_{1}\right)^{\check{\alpha}}}{\left(\eta_{2}-\eta_{1}\right)^{\check{\alpha}}} \mathcal{G}\left(\eta_{1}\right)-\frac{\Gamma(1+\check{\alpha})}{\left(\eta_{2}-\eta_{1}\right)^{\check{\alpha}} \eta_{1} \mathcal{I}_{y}^{(\check{\alpha})} \mathcal{G}(u)}
\end{aligned}
$$

and

$$
\begin{aligned}
& \frac{\left(\eta_{2}-y\right)^{2 \check{\alpha}}}{\left(\eta_{2}-\eta_{1}\right)^{\check{\alpha}}} \frac{1}{\Gamma(1+\check{\alpha})} \int_{0}^{1}(1-\rho)^{\check{\alpha}} \mathcal{G}^{(\check{\alpha})}\left(\rho y+(1-\rho) \eta_{2}\right)(d \rho)^{\check{\alpha}} \\
& =\frac{\left(\eta_{2}-y\right)^{2 \check{\alpha}}}{\left(\eta_{2}-\eta_{1}\right)^{\check{\alpha}}}\left[\left.\frac{(1-\rho)^{\check{\alpha}} \mathcal{G}\left(\rho y+(1-\rho) \eta_{2}\right)}{\left(y-\eta_{2}\right)^{\check{\alpha}}}\right|_{0} ^{1}\right. \\
& \left.\quad+\frac{1}{\left(y-\eta_{2}\right)^{\check{\alpha}}} \frac{\Gamma(1+\check{\alpha})}{\Gamma(1+\check{\alpha})} \int_{0}^{1} \mathcal{G}\left(\rho y+(1-\rho) \eta_{2}\right)(d \rho)^{\check{\alpha}}\right]
\end{aligned}
$$




$$
\begin{aligned}
& =\frac{\left(y-\eta_{2}\right)^{2 \check{\alpha}}}{\left(\eta_{2}-\eta_{1}\right)^{\check{\alpha}}}\left[(-1)^{\check{\alpha}} \frac{\mathcal{G}\left(\eta_{2}\right)}{\left(y-\eta_{2}\right)^{\check{\alpha}}}+\left(\frac{1}{y-\eta_{2}}\right)^{2 \check{\alpha}} \frac{\Gamma(1+\check{\alpha})}{\Gamma(1+\check{\alpha})} \int_{y}^{\eta_{2}} \mathcal{G}(u)(d u)^{\check{\alpha}}\right] \\
& =\frac{\left(\eta_{2}-y\right)^{\check{\alpha}}}{\left(\eta_{2}-\eta_{1}\right)^{\check{\alpha}}} \mathcal{G}\left(\eta_{2}\right)-\frac{\Gamma(1+\check{\alpha})}{\left(\eta_{2}-\eta_{1}\right)^{2 \check{\alpha}} y} \mathcal{I}_{\eta_{2}}^{(\check{\alpha})} \mathcal{G}(u) .
\end{aligned}
$$

By adding identities (3.2) and (3.3) we get the desired identity (3.1).

\section{Hermite-Hadamard-type inequalities for first-order differentiable functions}

Using Lemma 3.1, we now give some novel generalizations of the Hermite-Hadamardtype inequality for functions with generalized convex local derivatives.

Theorem 4.1 Let $\mathcal{G}: \Omega \rightarrow \mathbb{R}^{\check{\alpha}}(0<\check{\alpha} \leq 1)$ be such that $\mathcal{G} \in \mathcal{D}_{\check{\alpha}}(\Omega), \mathcal{G}^{(\check{\alpha})} \in \mathcal{C}_{\check{\alpha}}\left[\eta_{1}, \eta_{2}\right]$, and $\left|\mathcal{G}^{(\check{\alpha})}\right|$ is a generalized convex function on $\Omega$. Then

$$
\begin{aligned}
& \left|\frac{\left(y-\eta_{1}\right)^{\check{\alpha}} \mathcal{G}\left(\eta_{1}\right)+\left(\eta_{2}-y\right)^{\check{\alpha}} \mathcal{G}\left(\eta_{2}\right)}{\left(\eta_{2}-\eta_{1}\right)^{\check{\alpha}}}-\frac{\Gamma(1+\check{\alpha})}{\left(\eta_{2}-\eta_{1}\right)^{\check{\alpha}}} \eta_{1} \mathcal{I}_{\eta_{2}}^{(\check{\alpha})} \mathcal{G}(u)\right| \\
& \leq \frac{\left(y-\eta_{1}\right)^{2 \check{\alpha}}}{\left(\eta_{2}-\eta_{1}\right)^{\check{\alpha}}}\left[\left[\frac{\Gamma(1+\check{\alpha})}{\Gamma(1+2 \check{\alpha})}-\frac{\Gamma(1+2 \check{\alpha})}{\Gamma(1+3 \check{\alpha})}\right]\left|\mathcal{G}^{(\check{\alpha})}(y)\right|+\frac{\Gamma(1+2 \check{\alpha})}{\Gamma(1+3 \check{\alpha})}\left|\mathcal{G}^{(\check{\alpha})}\left(\eta_{1}\right)\right|\right] \\
& \quad+\frac{\left(\eta_{2}-y\right)^{2 \check{\alpha}}}{\left(\eta_{2}-\eta_{1}\right)^{\check{\alpha}}}\left[\left[\frac{\Gamma(1+\check{\alpha})}{\Gamma(1+2 \check{\alpha})}-\frac{\Gamma(1+2 \check{\alpha})}{\Gamma(1+3 \check{\alpha})}\right]\left|\mathcal{G}^{(\check{\alpha})}(y)\right|+\frac{\Gamma(1+2 \check{\alpha})}{\Gamma(1+3 \check{\alpha})}\left|\mathcal{G}^{(\check{\alpha})}\left(\eta_{2}\right)\right|\right]
\end{aligned}
$$

for all $y \in\left[\eta_{1}, \eta_{2}\right]$.

Proof It follows from Lemma 3.1 and the modulus property that

$$
\begin{aligned}
& \mid \frac{\left(y-\eta_{1}\right)^{\check{\alpha}} \mathcal{G}\left(\eta_{1}\right)+\left(\eta_{2}-y\right)^{\check{\alpha}} \mathcal{G}\left(\eta_{2}\right)}{\left(\eta_{2}-\eta_{1}\right)^{\check{\alpha}}}-\frac{\Gamma(1+\check{\alpha})}{\left(\eta_{2}-\eta_{1}\right)^{\check{\alpha}} \eta_{1}} \mathcal{I}_{\eta_{2}}^{(\check{\alpha})} \mathcal{G}(u) \mid \\
& \leq \frac{\left(y-\eta_{1}\right)^{2 \check{\alpha}}}{\left(\eta_{2}-\eta_{1}\right)^{\check{\alpha}}} \frac{1}{\Gamma(1+\check{\alpha})} \int_{0}^{1}(1-\rho)^{\check{\alpha}}\left|\mathcal{G}^{(\check{\alpha})}\left(\rho y+(1-\rho) \eta_{1}\right)\right|(d \rho)^{\check{\alpha}} \\
& \quad+\frac{\left(\eta_{2}-y\right)^{2 \check{\alpha}}}{\left(\eta_{2}-\eta_{1}\right)^{\check{\alpha}}} \frac{1}{\Gamma(1+\check{\alpha})} \int_{0}^{1}(1-\rho)^{\check{\alpha}}\left|\mathcal{G}^{(\check{\alpha})}\left(\rho y+(1-\rho) \eta_{2}\right)\right|(d \rho)^{\check{\alpha}} .
\end{aligned}
$$

Since $\left|\mathcal{G}^{(\breve{\alpha})}\right|$ is generalized convex, we get

$$
\begin{aligned}
& \left|\frac{\left(y-\eta_{1}\right)^{\check{\alpha}} \mathcal{G}\left(\eta_{1}\right)+\left(\eta_{2}-y\right)^{\check{\alpha}} \mathcal{G}\left(\eta_{2}\right)}{\left(\eta_{2}-\eta_{1}\right)^{\check{\alpha}}}-\frac{\Gamma(1+\check{\alpha})}{\left(\eta_{2}-\eta_{1}\right)^{\check{\alpha}} \eta_{1}} \mathcal{I}_{\eta_{2}}^{(\check{\alpha}} \mathcal{G}(u)\right| \\
& \leq \frac{\left(y-\eta_{1}\right)^{2 \check{\alpha}}}{\left(\eta_{2}-\eta_{1}\right)^{\check{\alpha}}}\left[\frac{1}{\Gamma(1+\check{\alpha})} \int_{0}^{1}(1-\rho)^{\check{\alpha}}\left[\rho^{\check{\alpha}}\left|\mathcal{G}^{(\check{\alpha})}(y)\right|+(1-\rho)^{\check{\alpha}}\left|\mathcal{G}^{(\check{\alpha})}\left(\eta_{1}\right)\right|\right](d \rho)^{\check{\alpha}}\right] \\
& \quad+\frac{\left(\eta_{2}-y\right)^{2 \check{\alpha}}}{\left(\eta_{2}-\eta_{1}\right)^{\check{\alpha}}}\left[\frac{1}{\Gamma(1+\check{\alpha})} \int_{0}^{1}(1-\rho)^{\check{\alpha}}\left[\rho^{\check{\alpha}}\left|\mathcal{G}^{(\check{\alpha})}(y)\right|+(1-\rho)^{\check{\alpha}}\left|\mathcal{G}^{(\check{\alpha})}\left(\eta_{2}\right)\right|\right](d \rho)^{\check{\alpha}}\right] \\
& =\frac{\left(y-\eta_{1}\right)^{2 \check{\alpha}}}{\left(\eta_{2}-\eta_{1}\right)^{\check{\alpha}}}\left[\left[\frac{\Gamma(1+\check{\alpha})}{\Gamma(1+2 \check{\alpha})}-\frac{\Gamma(1+2 \check{\alpha})}{\Gamma(1+3 \check{\alpha})}\right]\left|\mathcal{G}^{(\check{\alpha})}(y)\right|+\frac{\Gamma(1+2 \check{\alpha})}{\Gamma(1+3 \check{\alpha})}\left|\mathcal{G}^{(\check{\alpha})}\left(\eta_{1}\right)\right|\right] \\
& \quad+\frac{\left(\eta_{2}-y\right)^{2 \check{\alpha}}}{\left(\eta_{2}-\eta_{1}\right)^{\check{\alpha}}}\left[\left[\frac{\Gamma(1+\check{\alpha})}{\Gamma(1+2 \check{\alpha})}-\frac{\Gamma(1+2 \check{\alpha})}{\Gamma(1+3 \check{\alpha})}\right]\left|\mathcal{G}^{(\check{\alpha})}(y)\right|+\frac{\Gamma(1+2 \check{\alpha})}{\Gamma(1+3 \check{\alpha})}\left|\mathcal{G}^{(\check{\alpha})}\left(\eta_{2}\right)\right|\right],
\end{aligned}
$$


where we have used the equalities

$$
\begin{aligned}
& \frac{1}{\Gamma(1+\check{\alpha})} \int_{0}^{1} \rho^{\check{\alpha}}(1-\rho)^{\check{\alpha}}(d \rho)^{\check{\alpha}}=\frac{\Gamma(1+\check{\alpha})}{\Gamma(1+2 \check{\alpha})}-\frac{\Gamma(1+2 \check{\alpha})}{\Gamma(1+3 \check{\alpha})}, \\
& \frac{1}{\Gamma(1+\check{\alpha})} \int_{0}^{1}(1-\rho)^{2 \check{\alpha}}(d \rho)^{\check{\alpha}}=\frac{\Gamma(1+2 \check{\alpha})}{\Gamma(1+3 \check{\alpha})} .
\end{aligned}
$$

This completes the proof.

Some particular remarkable cases of Theorem 4.1 are as follows.

Corollary 4.2 Under the assumptions of Theorem 4.1, if we take $y=\frac{\eta_{1}+\eta_{2}}{2}$, then we have

$$
\begin{aligned}
& \mid \frac{\mathcal{G}\left(\eta_{1}\right)+\mathcal{G}\left(\eta_{2}\right)}{2^{\check{\alpha}}}-\frac{\Gamma(1+\check{\alpha})}{\left(\eta_{2}-\eta_{1}\right)^{\check{\alpha}} \eta_{1} \mathcal{I}_{\eta_{2}}^{(\check{\alpha})} \mathcal{G}(u) \mid} \\
& \leq \frac{\left(\eta_{2}-\eta_{1}\right)^{\check{\alpha}}}{4^{\check{\alpha}}}\left[\frac{\Gamma(1+2 \check{\alpha})}{\Gamma(1+3 \check{\alpha})}\left[\left|\mathcal{G}^{(\check{\alpha})}\left(\eta_{1}\right)\right|+\left|\mathcal{G}^{(\check{\alpha})}\left(\eta_{2}\right)\right|\right]\right. \\
& \left.\quad+2^{\check{\alpha}}\left[\frac{\Gamma(1+\check{\alpha})}{\Gamma(1+2 \check{\alpha})}-\frac{\Gamma(1+2 \check{\alpha})}{\Gamma(1+3 \check{\alpha})}\right]\left|\mathcal{G}^{(\check{\alpha})}\left(\frac{\eta_{1}+\eta_{2}}{2}\right)\right|\right] .
\end{aligned}
$$

Remark 4.3 Using the convexity of $\left|\mathcal{G}^{(\check{\alpha})}\right|$ in Corollary 4.2, we get

$$
\begin{aligned}
& \left|\frac{\mathcal{G}\left(\eta_{1}\right)+\mathcal{G}\left(\eta_{2}\right)}{2^{\check{\alpha}}}-\frac{\Gamma(1+\check{\alpha})}{\left(\eta_{2}-\eta_{1}\right)^{\check{\alpha}}} \eta_{1} \mathcal{I}_{\eta_{2}}^{(\check{\alpha})} \mathcal{G}(u)\right| \\
& \quad \leq \frac{\left(\eta_{2}-\eta_{1}\right)^{\check{\alpha}}}{4^{\check{\alpha}}} \frac{\Gamma(1+\check{\alpha})}{\Gamma(1+2 \check{\alpha})}\left[\left|\mathcal{G}^{(\check{\alpha})}\left(\eta_{1}\right)\right|+\left|\mathcal{G}^{(\check{\alpha})}\left(\eta_{2}\right)\right|\right] .
\end{aligned}
$$

Remark 4.4 If we choose $\check{\alpha}=1$, then Theorem 4.1, Corollary 4.2, and Remark 4.3 reduce to the results for classical convex functions given in $[23,24]$.

Theorem 4.5 Let $\rho, \omega>1$ with $\rho^{-1}+\omega^{-1}=1$, let $0<\check{\alpha} \leq 1$, and let a mapping $\mathcal{G}: \Omega \rightarrow \mathbb{R}^{\check{\alpha}}$ be such that $\mathcal{G} \in \mathcal{D}_{\check{\alpha}}(\Omega), \mathcal{G}^{(2 \check{\alpha})} \in \mathcal{C}_{\check{\alpha}}\left[\eta_{1}, \eta_{2}\right]$, and $\left|\mathcal{G}^{(2 \check{)})}\right|^{\rho}$ is a generalized convex function on $\Omega$. Then

$$
\begin{aligned}
& \left|\frac{\left(y-\eta_{1}\right)^{\check{\alpha}} \mathcal{G}\left(\eta_{1}\right)+\left(\eta_{2}-y\right)^{\check{\alpha}} \mathcal{G}\left(\eta_{2}\right)}{\left(\eta_{2}-\eta_{1}\right)^{\check{\alpha}}}-\frac{\Gamma(1+\check{\alpha})}{\left(\eta_{2}-\eta_{1}\right)^{\check{\alpha}} \eta_{1}} \mathcal{I}_{\eta_{2}}^{(\check{\alpha})} \mathcal{G}(u)\right| \\
& \leq\left(\frac{\Gamma(1+\omega \check{\alpha})}{\Gamma(1+(\omega+1) \check{\alpha})}\right)^{1 / \omega}\left[\frac{\Gamma(1+\check{\alpha})}{\Gamma(1+2 \check{\alpha})}\right]^{1 / \rho}\left[\frac{\left(y-\eta_{1}\right)^{2 \check{\alpha}}}{\left(\eta_{2}-\eta_{1}\right)^{\check{\alpha}}}\left[\left|\mathcal{G}^{(\check{\alpha})}(y)\right|^{\rho}+\left|\mathcal{G}^{(\check{\alpha})}\left(\eta_{1}\right)\right|^{\rho}\right]^{1 / \rho}\right. \\
& \left.\quad+\frac{\left(\eta_{2}-y\right)^{2 \check{\alpha}}}{\left(\eta_{2}-\eta_{1}\right)^{\check{\alpha}}}\left[\left|\mathcal{G}^{(\check{\alpha})}(y)\right|^{\rho}+\left|\mathcal{G}^{(\check{\alpha})}\left(\eta_{2}\right)\right|^{\rho}\right]^{1 / \rho}\right]
\end{aligned}
$$

for all $y \in\left[\eta_{1}, \eta_{2}\right]$.

Proof It follows from Lemma 3.1 and the generalized Hölder integral inequality that

$$
\begin{aligned}
& \left|\frac{\left(y-\eta_{1}\right)^{\check{\mathcal{G}}} \mathcal{G}\left(\eta_{1}\right)+\left(\eta_{2}-y\right)^{\check{\mathcal{G}}} \mathcal{G}\left(\eta_{2}\right)}{\left(\eta_{2}-\eta_{1}\right)^{\check{\alpha}}}-\frac{\Gamma(1+\check{\alpha})}{\left(\eta_{2}-\eta_{1}\right)^{\check{\alpha}} \eta_{1}} \mathcal{I}_{\eta_{2}}^{(\check{\alpha})} \mathcal{G}(u)\right| \\
& \quad \leq \frac{\left(y-\eta_{1}\right)^{2 \check{\alpha}}}{\left(\eta_{2}-\eta_{1}\right)^{\check{\alpha}}} \frac{1}{\Gamma(1+\check{\alpha})} \int_{0}^{1}(1-\rho)^{\check{\alpha}}\left|\mathcal{G}^{(\check{\alpha})}\left(\rho y+(1-\rho) \eta_{1}\right)\right|(d \rho)^{\check{\alpha}}
\end{aligned}
$$




$$
\begin{aligned}
& \quad+\frac{\left(\eta_{2}-y\right)^{2 \check{\alpha}}}{\left(\eta_{2}-\eta_{1}\right)^{\check{\alpha}}} \frac{1}{\Gamma(1+\check{\alpha})} \int_{0}^{1}(1-\rho)^{\check{\alpha}}\left|\mathcal{G}^{(\check{\alpha})}\left(\rho y+(1-\rho) \eta_{2}\right)\right|(d \rho)^{\check{\alpha}} \\
& \leq \frac{\left(y-\eta_{1}\right)^{2 \check{\alpha}}}{\left(\eta_{2}-\eta_{1}\right)^{\check{\alpha}}}\left(\frac{1}{\Gamma(1+\check{\alpha})} \int_{0}^{1}(1-\rho)^{\omega \check{\alpha}}(d \rho)^{\check{\alpha}}\right)^{1 / \omega} \\
& \quad \times\left(\frac{1}{\Gamma(1+\check{\alpha})} \int_{0}^{1}\left|\mathcal{G}^{(\check{\alpha})}\left(\rho y+(1-\rho) \eta_{1}\right)\right|^{\rho}(d \rho)^{\check{\alpha}}\right)^{1 / \rho} \\
& +\frac{\left(\eta_{2}-y\right)^{2 \check{\alpha}}}{\left(\eta_{2}-\eta_{1}\right)^{\check{\alpha}}}\left(\frac{1}{\Gamma(1+\check{\alpha})} \int_{0}^{1}(1-\rho)^{\omega \check{\alpha}}(d \rho)^{\check{\alpha}}\right)^{1 / \omega} \\
& \quad \times\left(\frac{1}{\Gamma(1+\check{\alpha})} \int_{0}^{1}\left|\mathcal{G}^{(\check{\alpha})}\left(\rho y+(1-\rho) \eta_{2}\right)\right|^{\rho}(d \rho)^{\check{\alpha}}\right)^{1 / \rho} .
\end{aligned}
$$

Since $\left|\mathcal{G}^{(\breve{\alpha})}\right|^{\rho}$ is a generalized convex function, we have

$$
\begin{aligned}
\left|\frac{\left(y-\eta_{1}\right)^{\check{\alpha}} \mathcal{G}\left(\eta_{1}\right)+\left(\eta_{2}-y\right)^{\check{\alpha}} \mathcal{G}\left(\eta_{2}\right)}{\left(\eta_{2}-\eta_{1}\right)^{\check{\alpha}}}-\frac{\Gamma(1+\check{\alpha})}{\left(\eta_{2}-\eta_{1}\right)^{\check{\alpha}} \eta_{1}} \mathcal{I}_{\eta_{2}}^{(\check{\alpha})} \mathcal{G}(u)\right| \\
\leq \frac{\left(y-\eta_{1}\right)^{2 \check{\alpha}}}{\left(\eta_{2}-\eta_{1}\right)^{\check{\alpha}}}\left(\frac{\Gamma(1+\omega \check{\alpha})}{\Gamma(1+(\omega+1) \check{\alpha})}\right)^{1 / \omega} \\
\quad \times\left[\frac{1}{\Gamma(1+\check{\alpha})} \int_{0}^{1}\left[\rho^{\check{\alpha}}\left|\mathcal{G}^{(\check{\alpha})}(y)\right|^{\rho}+(1-\rho)^{\check{\alpha}}\left|\mathcal{G}^{(\check{\alpha})}\left(\eta_{1}\right)\right|^{\rho}\right](d \rho)^{\check{\alpha}}\right]^{1 / \rho} \\
\quad+\frac{\left(\eta_{2}-y\right)^{2 \check{\alpha}}}{\left(\eta_{2}-\eta_{1}\right)^{\check{\alpha}}}\left(\frac{\Gamma(1+\omega \check{\alpha})}{\Gamma(1+(\omega+1) \check{\alpha})}\right)^{1 / \omega} \\
\quad \times\left[\frac{1}{\Gamma(1+\check{\alpha})} \int_{0}^{1}\left[\rho^{\check{\alpha}}\left|\mathcal{G}^{(\check{\alpha})}(y)\right|^{\rho}+(1-\rho)^{\check{\alpha}}\left|\mathcal{G}^{(\check{\alpha})}\left(\eta_{2}\right)\right|^{\rho}\right](d \rho)^{\check{\alpha}}\right]^{1 / \rho} \\
=\frac{\left(y-\eta_{1}\right)^{2 \check{\alpha}}}{\left(\eta_{2}-\eta_{1}\right)^{\check{\alpha}}}\left(\frac{\Gamma(1+\omega \check{\alpha})}{\Gamma(1+(\omega+1) \check{\alpha})}\right)^{1 / \omega}\left[\frac{\Gamma(1+\check{\alpha})}{\Gamma(1+2 \check{\alpha})}\left[\left|\mathcal{G}^{(\check{\alpha})}(y)\right|^{\rho}+\left|\mathcal{G}^{(\check{\alpha})}\left(\eta_{1}\right)\right|^{\rho}\right]^{1 / \rho}\right] \\
\quad+\frac{\left(\eta_{2}-y\right)^{2 \check{\alpha}}}{\left(\eta_{2}-\eta_{1}\right)^{\check{\alpha}}}\left(\frac{\Gamma(1+\omega \check{\alpha})}{\Gamma(1+(\omega+1) \check{\alpha})}\right)^{1 / \omega} \\
\quad \times\left[\frac{\Gamma(1+\check{\alpha})}{\Gamma(1+2 \check{\alpha})}\left[\left|\mathcal{G}^{(\check{\alpha})}(y)\right|^{\rho}+\left|\mathcal{G}^{(\check{\alpha})}\left(\eta_{2}\right)\right|^{\rho}\right]^{1 / \rho}\right],
\end{aligned}
$$

where we have used the equality

$$
\frac{1}{\Gamma(1+\check{\alpha})} \int_{0}^{1}(1-\rho)^{\omega \check{\alpha}}(d \rho)^{\check{\alpha}}=\frac{\Gamma(1+\omega \check{\alpha})}{\Gamma(1+(\omega+1) \check{\alpha})} .
$$

This completes the proof.

Some particular cases of Theorem 4.5 and corollaries are as follows.

Corollary 4.6 Under the assumptions of Theorem 4.5, if we take $y=\frac{\eta_{1}+\eta_{2}}{2}$, then we have

$$
\begin{aligned}
& \left|\frac{\mathcal{G}\left(\eta_{1}\right)+\mathcal{G}\left(\eta_{2}\right)}{2^{\check{\alpha}}}-\frac{\Gamma(1+\check{\alpha})}{\left(\eta_{2}-\eta_{1}\right)^{\check{\alpha}} \eta_{1}} \mathcal{I}_{\eta_{2}}^{(\check{\alpha})} \mathcal{G}(u)\right| \\
& \quad \leq\left(\frac{\eta_{2}-\eta_{1}}{4}\right)^{\check{\alpha}}\left(\frac{\Gamma(1+\omega \check{\alpha})}{\Gamma(1+(\omega+1) \check{\alpha})}\right)^{1 / \omega}\left(\frac{\Gamma(1+\check{\alpha})}{\Gamma(1+2 \check{\alpha})}\right)^{1 / \rho}
\end{aligned}
$$




$$
\begin{aligned}
& \times\left[\left.|| \mathcal{G}^{(\check{\alpha})}\left(\frac{\eta_{1}+\eta_{2}}{2}\right)\right|^{\rho}+\left|\mathcal{G}^{(\check{\alpha})}\left(\eta_{1}\right)\right|^{\rho}\right]^{1 / \rho} \\
& \left.+\left[\left|\mathcal{G}^{(\check{\alpha})}\left(\frac{\eta_{1}+\eta_{2}}{2}\right)\right|^{\rho}+\left|\mathcal{G}^{(\check{\alpha})}\left(\eta_{2}\right)\right|^{\rho}\right]^{1 / \rho}\right] \\
\leq & \left(\frac{\eta_{2}-\eta_{1}}{2}\right)^{\check{\alpha}}\left(\frac{\Gamma(1+\omega \check{\alpha})}{\Gamma(1+(\omega+1) \check{\alpha})}\right)^{1 / \omega}\left(\frac{\Gamma(1+\check{\alpha})}{\Gamma(1+2 \check{\alpha})}\right)^{1 / \rho}\left[\left|\mathcal{G}^{(\check{\alpha})}\left(\eta_{1}\right)\right|+\left|\mathcal{G}^{(\check{\alpha})}\left(\eta_{2}\right)\right|\right],
\end{aligned}
$$

where in the second inequality, we used the inequality

$$
\sum_{i=1}^{p}\left(\mu_{i}+v_{i}\right)^{\kappa} \leq \sum_{i=1}^{p}\left(\mu_{i}\right)^{\kappa}+\sum_{i=1}^{p}\left(v_{i}\right)^{\kappa} \quad\left(0 \leq \kappa<1, \mu_{i}, v_{i} \geq 0, i=1, \ldots, p\right) .
$$

Theorem 4.7 Let $\rho, \omega>1$ with $\rho^{-1}+\omega^{-1}=1$, let $0<\check{\alpha} \leq 1$, and let a mapping $\mathcal{G}: \Omega \rightarrow \mathbb{R}^{\check{\alpha}}$ be such that $\mathcal{G} \in \mathcal{D}_{\check{\alpha}}(\Omega), \mathcal{G}^{(\breve{\alpha})} \in \mathcal{C}_{\breve{\alpha}}\left[\eta_{1}, \eta_{2}\right]$, and $\left|\mathcal{G}^{(\breve{\alpha})}\right|^{\rho}$ is a generalized convex function on $\Omega$. Then

$$
\begin{aligned}
& \left|\frac{\left(y-\eta_{1}\right)^{\check{\alpha}} \mathcal{G}\left(\eta_{1}\right)+\left(\eta_{2}-y\right)^{\check{\alpha}} \mathcal{G}\left(\eta_{2}\right)}{\left(\eta_{2}-\eta_{1}\right)^{\check{\alpha}}}-\frac{\Gamma(1+\check{\alpha})}{\left(\eta_{2}-\eta_{1}\right)^{\check{\alpha}} \eta_{1}} \mathcal{I}_{\eta_{2}}^{(\check{\alpha})} \mathcal{G}(u)\right| \\
& \leq\left(\frac{\Gamma(1+\check{\alpha})}{\Gamma(1+2 \check{\alpha})}\right)^{1-\frac{1}{\rho}}\left[\frac { ( \eta _ { 2 } - y ) ^ { 2 \check { \alpha } } } { ( \eta _ { 2 } - \eta _ { 1 } ) ^ { \check { \alpha } } } \left(\left[\frac{\Gamma(1+\check{\alpha})}{\Gamma(1+2 \check{\alpha})}-\frac{\Gamma(1+2 \check{\alpha})}{\Gamma(1+3 \check{\alpha})}\right]\left|\mathcal{G}^{(\check{\alpha})}(y)\right|^{\rho}\right.\right. \\
& \left.\quad+\frac{\Gamma(1+2 \check{\alpha})}{\Gamma(1+3 \check{\alpha})}\left|\mathcal{G}^{(\check{\alpha})}\left(\eta_{1}\right)\right|^{\rho}\right)^{1 / \rho} \\
& \quad+\frac{\left(y-\eta_{1}\right)^{2 \check{\alpha}}}{\left(\eta_{2}-\eta_{1}\right)^{\check{\alpha}}}\left(\left[\frac{\Gamma(1+\check{\alpha})}{\Gamma(1+2 \check{\alpha})}-\frac{\Gamma(1+2 \check{\alpha})}{\Gamma(1+3 \check{\alpha})}\right]\left|\mathcal{G}^{(\check{\alpha})}(y)\right|^{\rho}\right. \\
& \left.\left.\quad+\frac{\Gamma(1+2 \check{\alpha})}{\Gamma(1+3 \check{\alpha})}\left|\mathcal{G}^{(\check{\alpha})}\left(\eta_{2}\right)\right|^{\rho}\right)^{1 / \rho}\right]
\end{aligned}
$$

for all $y \in\left[\eta_{1}, \eta_{2}\right]$

Proof It follows from Lemma 3.1 and the generalized power-mean inequality that

$$
\begin{aligned}
&\left|\frac{\left(y-\eta_{1}\right)^{\check{\alpha}} \mathcal{G}\left(\eta_{1}\right)+\left(\eta_{2}-y\right)^{\check{\alpha}} \mathcal{G}\left(\eta_{2}\right)}{\left(\eta_{2}-\eta_{1}\right)^{\check{\alpha}}}-\frac{\Gamma(1+\check{\alpha})}{\left(\eta_{2}-\eta_{1}\right)^{\check{\alpha}} \eta_{1}} \mathcal{I}_{\eta_{2}}^{(\check{\alpha})} \mathcal{G}(u)\right| \\
& \leq \frac{\left(y-\eta_{1}\right)^{2 \check{\alpha}}}{\left(\eta_{2}-\eta_{1}\right)^{\check{\alpha}}} \frac{1}{\Gamma(1+\check{\alpha})} \int_{0}^{1}(1-\rho)^{\check{\alpha}}\left|\mathcal{G}^{(\check{\alpha})}\left(\rho y+(1-\rho) \eta_{1}\right)\right|(d \rho)^{\check{\alpha}} \\
& \quad+\frac{\left(\eta_{2}-y\right)^{2 \check{\alpha}}}{\left(\eta_{2}-\eta_{1}\right)^{\check{\alpha}}} \frac{1}{\Gamma(1+\check{\alpha})} \int_{0}^{1}(1-\rho)^{\check{\alpha}}\left|\mathcal{G}^{(\check{\alpha})}\left(\rho y+(1-\rho) \eta_{2}\right)\right|(d \rho)^{\check{\alpha}} \\
& \leq \frac{\left(y-\eta_{1}\right)^{2 \check{\alpha}}}{\left(\eta_{2}-\eta_{1}\right)^{\check{\alpha}}}\left(\frac{1}{\Gamma(1+\check{\alpha})} \int_{0}^{1}(1-\rho)^{\check{\alpha}}(d \rho)^{\check{\alpha}}\right)^{1-\frac{1}{\rho}} \\
& \quad \times\left(\frac{1}{\Gamma(1+\check{\alpha})} \int_{0}^{1}(1-\rho)^{\check{\alpha}}\left|\mathcal{G}^{(\check{\alpha})}\left(\rho y+(1-\rho) \eta_{1}\right)\right|^{\rho}(d \rho)^{\check{\alpha}}\right)^{1 / \rho} \\
& \quad+\frac{\left(\eta_{2}-y\right)^{2 \check{\alpha}}}{\left(\eta_{2}-\eta_{1}\right)^{\check{\alpha}}}\left(\frac{1}{\Gamma(1+\check{\alpha})} \int_{0}^{1}(1-\rho)^{\check{\alpha}}(d \rho)^{\check{\alpha}}\right)^{1-\frac{1}{\rho}} \\
& \quad \times\left(\frac{1}{\Gamma(1+\check{\alpha})} \int_{0}^{1}(1-\rho)^{\check{\alpha}}\left|\mathcal{G}^{(\check{\alpha})}\left(\rho y+(1-\rho) \eta_{2}\right)\right|^{\rho}(d \rho)^{\check{\alpha}}\right)^{1 / \rho} .
\end{aligned}
$$


Since $\left|\mathcal{G}^{(\check{\alpha})}\right|^{\rho}$ is a generalized convex function, we obtain

$$
\begin{aligned}
& \frac{1}{\Gamma(1+\check{\alpha})} \int_{0}^{1}(1-\rho)^{\check{\alpha}}\left|\mathcal{G}^{(\check{\alpha})}\left(\rho y+(1-\rho) \eta_{1}\right)\right|^{\rho}(d \rho)^{\check{\alpha}} \\
& \quad \leq \frac{1}{\Gamma(1+\check{\alpha})} \int_{0}^{1}(1-\rho)^{\check{\alpha}}\left[\rho^{(\check{\alpha})}\left|\mathcal{G}^{(\check{\alpha})}(y)\right|^{\rho}+(1-\rho)^{(\check{\alpha})}\left|\mathcal{G}^{(\check{\alpha})}\left(\eta_{1}\right)\right|^{\rho}\right](d \rho)^{\check{\alpha}} \\
& \quad=\left[\frac{\Gamma(1+\check{\alpha})}{\Gamma(1+2 \check{\alpha})}-\frac{\Gamma(1+2 \check{\alpha})}{\Gamma(1+3 \check{\alpha})}\right]\left|\mathcal{G}^{(\check{\alpha})}(y)\right|^{\rho}+\frac{\Gamma(1+2 \check{\alpha})}{\Gamma(1+3 \check{\alpha})}\left|\mathcal{G}^{(\check{\alpha})}\left(\eta_{1}\right)\right|^{\rho} .
\end{aligned}
$$

Similarly,

$$
\begin{aligned}
& \frac{1}{\Gamma(1+\check{\alpha})} \int_{0}^{1}(1-\rho)^{\check{\alpha}}\left|\mathcal{G}^{(\check{\alpha})}\left(\rho y+(1-\rho) \eta_{2}\right)\right|^{\rho}(d \rho)^{\check{\alpha}} \\
& \quad \leq\left[\frac{\Gamma(1+\check{\alpha})}{\Gamma(1+2 \check{\alpha})}-\frac{\Gamma(1+2 \check{\alpha})}{\Gamma(1+3 \check{\alpha})}\right]\left|\mathcal{G}^{(\check{\alpha})}(y)\right|^{\rho}+\frac{\Gamma(1+2 \check{\alpha})}{\Gamma(1+3 \check{\alpha})}\left|\mathcal{G}^{(\check{\alpha})}\left(\eta_{2}\right)\right|^{\rho} .
\end{aligned}
$$

Combining (4.6)-(4.8) gives the desired inequality (4.5). This completes the proof.

Corollary 4.8 Under the assumptions of Theorem 4.7, if we take $y=\frac{\eta_{1}+\eta_{2}}{2}$, then we have

$$
\begin{aligned}
& \left|\frac{\mathcal{G}\left(\eta_{1}\right)+\mathcal{G}\left(\eta_{2}\right)}{2^{\check{\alpha}}}-\frac{\Gamma(1+\check{\alpha})}{\left(\eta_{2}-\eta_{1}\right)^{\check{\alpha}} \eta_{1}} \mathcal{I}_{\eta_{2}}^{(\check{\alpha})} \mathcal{G}(u)\right| \\
& \leq\left(\frac{\eta_{2}-\eta_{1}}{4}\right)^{\check{\alpha}}\left(\frac{\Gamma(1+\check{\alpha})}{\Gamma(1+2 \check{\alpha})}\right)^{1-\frac{1}{\rho}} \\
& \quad \times\left[\left(\left[\frac{\Gamma(1+\check{\alpha})}{\Gamma(1+2 \check{\alpha})}-\frac{\Gamma(1+2 \check{\alpha})}{\Gamma(1+3 \check{\alpha})}\right]\left|\mathcal{G}^{(\check{\alpha})}\left(\frac{\eta_{1}+\eta_{2}}{2}\right)\right|^{\rho}+\frac{\Gamma(1+2 \check{\alpha})}{\Gamma(1+3 \check{\alpha})}\left|\mathcal{G}^{(\check{\alpha})}\left(\eta_{1}\right)\right|^{\rho}\right)^{1 / \rho}\right. \\
& \left.\quad+\left(\left[\frac{\Gamma(1+\check{\alpha})}{\Gamma(1+2 \check{\alpha})}-\frac{\Gamma(1+2 \check{\alpha})}{\Gamma(1+3 \check{\alpha})}\right]\left|\mathcal{G}^{(\check{\alpha})}\left(\frac{\eta_{1}+\eta_{2}}{2}\right)\right|^{\rho}+\frac{\Gamma(1+2 \check{\alpha})}{\Gamma(1+3 \check{\alpha})}\left|\mathcal{G}^{(\check{\alpha})}\left(\eta_{2}\right)\right|^{\rho}\right)^{1 / \rho}\right] .
\end{aligned}
$$

\section{Generalized inequalities for second-order differentiability}

This section is devoted to certain generalizations for twice locally differentiable functions, which are connected with the Hermite-Hadamard-type inequality. For this purpose, we need the following lemma.

Lemma 5.1 Let $\check{\alpha} \in(0,1]$, and let a mapping $\mathcal{G}: \Omega \rightarrow \mathbb{R}^{\check{\alpha}}$ be such that $\mathcal{G} \in \mathcal{D}_{\check{\alpha}}(\Omega)$ and $\mathcal{G}^{(2 \check{\alpha})} \in \mathcal{C}_{\check{\alpha}}\left[\eta_{1}, \eta_{2}\right]$. Then

$$
\begin{gathered}
{\left[\frac{\mathcal{G}\left(\eta_{1}\right)+\mathcal{G}\left(\eta_{2}\right)}{2^{\check{\alpha}}}\right] \Gamma(1+\check{\alpha})-\left(\frac{1}{\eta_{2}-\eta_{1}}\right)^{\check{\alpha}} \Gamma^{2}(1+\check{\alpha})_{\eta_{1}} \mathcal{I}_{\eta_{2}}^{(\check{\alpha})} \mathcal{G}(u)} \\
=\frac{\left(\eta_{2}-\eta_{1}\right)^{2 \check{\alpha}}}{16^{\check{\alpha}}}\left[\frac{1}{\Gamma(1+\check{\alpha})} \int_{0}^{1}\left(1-\vartheta^{2 \check{\alpha}}\right) \mathcal{G}^{(2 \check{\alpha})}\left(\frac{1-\vartheta}{2} \eta_{1}+\frac{1+\vartheta}{2} \eta_{2}\right)(d \vartheta)^{\check{\alpha}}\right. \\
\left.+\frac{1}{\Gamma(1+\check{\alpha})} \int_{0}^{1}\left(1-\vartheta^{2 \check{\alpha}}\right) \mathcal{G}^{(2 \check{\alpha})}\left(\frac{1+\vartheta}{2} \eta_{1}+\frac{1-\vartheta}{2} \eta_{2}\right)(d \vartheta)^{\check{\alpha}}\right] .
\end{gathered}
$$


Proof Using local fractional integration by parts and change of variable, we have

$$
\begin{aligned}
& \frac{1}{\Gamma(1+\check{\alpha})} \int_{0}^{1}\left(1-\vartheta^{2 \check{\alpha}}\right) \mathcal{G}^{(2 \check{\alpha})}\left(\frac{1+\vartheta}{2} \eta_{1}+\frac{1-\vartheta}{2} \eta_{2}\right)(d \vartheta)^{\check{\alpha}} \\
&=\left.\left(\frac{2}{\eta_{1}-\eta_{2}}\right)^{\check{\alpha}}\left(1-\vartheta^{2 \check{\alpha}}\right) \mathcal{G}^{(\check{\alpha})}\left(\frac{1+\vartheta}{2} \eta_{1}+\frac{1-\vartheta}{2} \eta_{2}\right)\right|_{0} ^{1} \\
& \quad-\left(\frac{4}{\eta_{2}-\eta_{1}}\right)^{\check{\alpha}} \frac{\Gamma(1+\check{\alpha})}{\Gamma(1+\check{\alpha})} \int_{0}^{1} \vartheta^{\check{\alpha}} \mathcal{G}^{\check{\alpha})}\left(\frac{1+\vartheta}{2} \eta_{1}+\frac{1-\vartheta}{2} \eta_{2}\right)(d \vartheta)^{\check{\alpha}} \\
&=\left(\frac{2}{\eta_{2}-\eta_{1}}\right)^{\check{\alpha}} \mathcal{G}^{(\check{\alpha})}\left(\frac{\eta_{1}+\eta_{2}}{2}\right)+\left(\frac{8}{\left(\eta_{2}-\eta_{1}\right)^{2}}\right)^{\check{\alpha}} \Gamma(1+\check{\alpha}) \mathcal{G}\left(\eta_{1}\right) \\
& \quad-\left(\frac{8}{\left(\eta_{2}-\eta_{1}\right)^{2}}\right)^{\check{\alpha}} \frac{\Gamma^{2}(1+\check{\alpha})}{\Gamma(1+\check{\alpha})} \int_{0}^{1} \mathcal{G}\left(\frac{1+\vartheta}{2} \eta_{1}+\frac{1-\vartheta}{2} \eta_{2}\right)(d \vartheta)^{\check{\alpha}} \\
&=\left.\frac{2}{\eta_{2}-\eta_{1}}\right)^{\check{\alpha}} \mathcal{G}^{(\check{\alpha})}\left(\frac{\eta_{1}+\eta_{2}}{2}\right)+\left(\frac{8}{\left(\eta_{2}-\eta_{1}\right)^{2}}\right)^{\check{\alpha}} \Gamma(1+\check{\alpha}) \mathcal{G}\left(\eta_{1}\right) \\
&-\left(\frac{16}{\left(\eta_{2}-\eta_{1}\right)^{3}}\right)^{\check{\alpha}} \frac{\Gamma^{2}(1+\check{\alpha})}{\Gamma(1+\check{\alpha})} \int_{\eta_{1}}^{\frac{\eta_{1}+\eta_{2}}{2}} \mathcal{G}(u)(d u)^{\check{\alpha}} \\
&=\left(\frac{2}{\eta_{2}-\eta_{1}}\right)^{\check{\alpha}} \mathcal{G}^{\check{\alpha})}\left(\frac{\eta_{1}+\eta_{2}}{2}\right)+\left(\frac{8}{\left(\eta_{2}-\eta_{1}\right)^{2}}\right)^{\check{\alpha}} \Gamma(1+\check{\alpha}) \mathcal{G}\left(\eta_{1}\right) \\
&-\left(\frac{16}{\left(\eta_{2}-\eta_{1}\right)^{3}}\right)^{\check{\alpha}} \Gamma^{2}(1+\check{\alpha})_{\eta_{1}} \mathcal{I}_{\frac{\left(\check{\alpha}+\eta_{2}\right.}{2} \mathcal{G}(u)}
\end{aligned}
$$

and, analogously,

$$
\begin{aligned}
& \frac{1}{\Gamma(1+\check{\alpha})} \int_{0}^{1}\left(1-\vartheta^{2 \check{\alpha}}\right) \mathcal{G}^{(2 \check{\alpha})}\left(\frac{1-\vartheta}{2} \eta_{1}+\frac{1+\vartheta}{2} \eta_{2}\right)(d \vartheta)^{\check{\alpha}} \\
&=-\left.\left(\frac{2}{\eta_{2}-\eta_{1}}\right)^{\check{\alpha}}\left(1-\vartheta^{2 \check{\alpha}}\right) \mathcal{G}^{(\check{\alpha})}\left(\frac{1-\vartheta}{2} \eta_{1}+\frac{1+\vartheta}{2} \eta_{2}\right)\right|_{0} ^{1} \\
&+\left(\frac{4}{\eta_{2}-\eta_{1}}\right)^{\check{\alpha}} \frac{\Gamma(1+\check{\alpha})}{\Gamma(1+\check{\alpha})} \int_{0}^{1} \vartheta^{\check{\alpha}} \mathcal{G}^{(\check{\alpha})}\left(\frac{1-\vartheta}{2} \eta_{1}+\frac{1+\vartheta}{2} \eta_{2}\right)(d \vartheta)^{\check{\alpha}} \\
&=-\left(\frac{2}{\eta_{2}-\eta_{1}}\right)^{\check{\alpha}} \mathcal{G}^{(\check{\alpha})}\left(\frac{\eta_{1}+\eta_{2}}{2}\right)+\left(\frac{8}{\left(\eta_{2}-\eta_{1}\right)^{2}}\right)^{\check{\alpha}} \Gamma(1+\check{\alpha}) \mathcal{G}\left(\eta_{2}\right) \\
&-\left(\frac{8}{\left(\eta_{2}-\eta_{1}\right)^{2}}\right)^{\check{\alpha}} \frac{\Gamma^{2}(1+\check{\alpha})}{\Gamma(1+\check{\alpha})} \int_{0}^{1} \mathcal{G}\left(\frac{1-\vartheta}{2} \eta_{1}+\frac{1+\vartheta}{2} \eta_{2}\right)(d \vartheta)^{\check{\alpha}} \\
&=-\left(\frac{2}{\eta_{2}-\eta_{1}}\right)^{\check{\alpha}} \mathcal{G}^{(\check{\alpha})}\left(\frac{\eta_{1}+\eta_{2}}{2}\right)+\left(\frac{8}{\left(\eta_{2}-\eta_{1}\right)^{2}}\right)^{\check{\alpha}} \Gamma(1+\check{\alpha}) \mathcal{G}\left(\eta_{2}\right) \\
&-\left(\frac{16}{\left(\eta_{2}-\eta_{1}\right)^{3}}\right)^{\check{\alpha}} \frac{\Gamma^{2}(1+\check{\alpha})}{\Gamma(1+\check{\alpha})} \int_{\frac{\eta_{1}+\eta_{2}}{2}}^{\eta_{2}} \mathcal{G}(u)(d u)^{\check{\alpha}} \\
&=-\left(\frac{2}{\eta_{2}-\eta_{1}}\right)^{\check{\alpha}} \mathcal{G}^{(\check{\alpha})}\left(\frac{\eta_{1}+\eta_{2}}{2}\right)+\left(\frac{8}{\left(\eta_{2}-\eta_{1}\right)^{2}}\right)^{\check{\alpha}} \Gamma(1+\check{\alpha}) \mathcal{G}\left(\eta_{2}\right) \\
&-\left(\frac{16}{\left(\eta_{2}-\eta_{1}\right)^{3}}\right)^{\check{\alpha}} \Gamma^{2}(1+\check{\alpha})_{\frac{\eta_{1}+\eta_{2}}{2}} \mathcal{I}_{\eta_{2}}^{(\check{\alpha})} \mathcal{G}(u) .
\end{aligned}
$$


Adding (5.2) and (5.3) and then multiplying both sides by $\left(\frac{\left(\eta_{2}-\eta_{1}\right)^{2}}{16}\right)^{\check{\alpha}}$ give the desired identity. This completes the proof.

Remark 5.2 If we take $\check{\alpha}=1$, then Lemma 5.1 reduces to the result presented by Barani et al. [25].

Theorem 5.3 Let a mapping $\mathcal{G}: \Omega \rightarrow \mathbb{R}^{\check{\alpha}}(0<\check{\alpha} \leq 1)$ be such that $\mathcal{G} \in \mathcal{D}_{\check{\alpha}}(\Omega)$, $\mathcal{G}^{(2 \check{\alpha})} \in$ $\mathcal{C}_{\check{\alpha}}\left[\eta_{1}, \eta_{2}\right]$, and $\left|\mathcal{G}^{(2 \check{\alpha})}\right|$ is a generalized convex function on $\Omega$. Then

$$
\begin{aligned}
& \left|\left[\frac{\mathcal{G}\left(\eta_{1}\right)+\mathcal{G}\left(\eta_{2}\right)}{2^{\check{\alpha}}}\right] \Gamma(1+\check{\alpha})-\left(\frac{1}{\eta_{2}-\eta_{1}}\right)^{\check{\alpha}} \Gamma^{2}(1+\check{\alpha})_{\eta_{1}} \mathcal{I}_{\eta_{2}}^{(\check{\alpha})} \mathcal{G}(u)\right| \\
& \quad \leq \frac{\left(\eta_{2}-\eta_{1}\right)^{2 \check{\alpha}}}{16^{\check{\alpha}}}\left[\left|\mathcal{G}^{(2 \check{\alpha})}\left(\eta_{1}\right)\right|+\left|\mathcal{G}^{(2 \check{\alpha})}\left(\eta_{2}\right)\right|\right]\left[\frac{1}{\Gamma(1+\check{\alpha})}-\frac{\Gamma(1+2 \check{\alpha})}{\Gamma(1+3 \check{\alpha})}\right] .
\end{aligned}
$$

Proof From Lemma 5.1 and the modulus property we have

$$
\begin{aligned}
& \left|\left[\frac{\mathcal{G}\left(\eta_{1}\right)+\mathcal{G}\left(\eta_{2}\right)}{2^{\check{\alpha}}}\right] \Gamma(1+\check{\alpha})-\left(\frac{1}{\eta_{2}-\eta_{1}}\right)^{\check{\alpha}} \Gamma^{2}(1+\check{\alpha})_{\eta_{1}} \mathcal{I}_{\eta_{2}}^{(\check{\alpha})} \mathcal{G}(u)\right| \\
& \quad \leq \frac{\left(\eta_{2}-\eta_{1}\right)^{2 \check{\alpha}}}{16^{\check{\alpha}}}\left[\frac{1}{\Gamma(1+\check{\alpha})} \int_{0}^{1}\left(1-\vartheta^{2 \check{\alpha}}\right)\left|\mathcal{G}^{(2 \check{\alpha})}\left(\frac{1-\vartheta}{2} \eta_{1}+\frac{1+\vartheta}{2} \eta_{2}\right)\right|(d \vartheta)^{\check{\alpha}}\right. \\
& \left.\quad+\frac{1}{\Gamma(1+\check{\alpha})} \int_{0}^{1}\left(1-\vartheta^{2 \check{\alpha}}\right)\left|\mathcal{G}^{(2 \check{\alpha})}\left(\frac{1+\vartheta}{2} \eta_{1}+\frac{1-\vartheta}{2} \eta_{2}\right)\right|(d \vartheta)^{\check{\alpha}}\right] .
\end{aligned}
$$

Since $\left|\mathcal{G}^{(2 \check{\alpha})}\right|$ is a generalized convex function on $\Omega$, we have

$$
\left|\mathcal{G}^{(2 \check{\alpha})}\left(\frac{1-\vartheta}{2} \eta_{1}+\frac{1+\vartheta}{2} \eta_{2}\right)\right| \leq\left(\frac{1-\vartheta}{2}\right)^{\check{\alpha}}\left|\mathcal{G}^{(2 \check{\alpha})}\left(\eta_{1}\right)\right|+\left(\frac{1+\vartheta}{2}\right)^{\check{\alpha}}\left|\mathcal{G}^{(2 \check{\alpha})}\left(\eta_{2}\right)\right|
$$

and

$$
\left|\mathcal{G}^{(2 \check{\alpha})}\left(\frac{1+\vartheta}{2} \eta_{1}+\frac{1-\vartheta}{2} \eta_{2}\right)\right| \leq\left(\frac{1+\vartheta}{2}\right)^{\check{\alpha}}\left|\mathcal{G}^{(2 \check{\alpha})}\left(\eta_{1}\right)\right|+\left(\frac{1-\vartheta}{2}\right)^{\check{\alpha}}\left|\mathcal{G}^{(2 \check{\alpha})}\left(\eta_{2}\right)\right| .
$$

Therefore

$$
\begin{aligned}
& \left|\left[\frac{\mathcal{G}\left(\eta_{1}\right)+\mathcal{G}\left(\eta_{2}\right)}{2^{\check{\alpha}}}\right] \Gamma(1+\check{\alpha})-\left(\frac{1}{\eta_{2}-\eta_{1}}\right)^{\check{\alpha}} \Gamma^{2}(1+\check{\alpha})_{\eta_{1}} \mathcal{I}_{\eta_{2}}^{(\check{\alpha})} \mathcal{G}(u)\right| \\
& \leq \frac{\left(\eta_{2}-\eta_{1}\right)^{2 \check{\alpha}}}{16^{\check{\alpha}}}\left[\frac{1}{\Gamma(1+\check{\alpha})}\right. \\
& \quad \times \int_{0}^{1}\left(1-\vartheta^{2 \check{\alpha}}\right)\left\{\left(\frac{1-\vartheta}{2}\right)^{\check{\alpha}}\left|\mathcal{G}^{(2 \check{\alpha})}\left(\eta_{1}\right)\right|+\left(\frac{1+\vartheta}{2}\right)^{\check{\alpha}}\left|\mathcal{G}^{(2 \check{\alpha})}\left(\eta_{2}\right)\right|\right\}(d \vartheta)^{\check{\alpha}} \\
& \left.\quad+\frac{1}{\Gamma(1+\check{\alpha})} \int_{0}^{1}\left(1-\vartheta^{2 \check{\alpha}}\right)\left\{\left(\frac{1+\vartheta}{2}\right)^{\check{\alpha}}\left|\mathcal{G}^{(2 \check{\alpha})}\left(\eta_{1}\right)\right|+\left(\frac{1-\vartheta}{2}\right)^{\check{\alpha}}\left|\mathcal{G}^{(2 \check{\alpha})}\left(\eta_{2}\right)\right|\right\}(d \vartheta)^{\check{\alpha}}\right] \\
& \leq \frac{\left(\eta_{2}-\eta_{1}\right)^{2 \check{\alpha}}}{32^{\check{\alpha}}}\left[\left|\mathcal{G}^{(2 \check{\alpha})}\left(\eta_{1}\right)\right|+\left|\mathcal{G}^{(2 \check{\alpha})}\left(\eta_{2}\right)\right|\right] \\
& \quad \times\left[\frac{1}{\Gamma(1+\check{\alpha})} \int_{0}^{1}\left\{\left(1-t^{2 \check{\alpha}}\right)\left[(1-\vartheta)^{\check{\alpha}}+(1+\vartheta)^{\check{\alpha}}\right]\right\}(d \vartheta)^{\check{\alpha}}\right]
\end{aligned}
$$


where we have used the equality

$$
\begin{aligned}
& \frac{1}{\Gamma(1+\check{\alpha})} \int_{0}^{1}\left\{\left(1-\vartheta^{2 \check{\alpha}}\right)\left[(1-\vartheta)^{\check{\alpha}}+(1+\vartheta)^{\check{\alpha}}\right]\right\}(d \vartheta)^{\check{\alpha}} \\
& \quad=2^{\check{\alpha}}\left[\frac{1}{\Gamma(1+\check{\alpha})}-\frac{\Gamma(1+2 \check{\alpha})}{\Gamma(1+3 \check{\alpha})}\right] .
\end{aligned}
$$

This completes the proof.

Theorem 5.4 Let $\rho, \omega>1$ with $\rho^{-1}+\omega^{-1}=1$, and let a mapping $\mathcal{G}: \Omega \rightarrow \mathbb{R}^{\check{\alpha}}(0<\check{\alpha} \leq 1)$ be such that $\mathcal{G} \in \mathcal{D}_{\check{\alpha}}(\Omega), \mathcal{G}^{(2 \check{\alpha})} \in \mathcal{C}_{\check{\alpha}}\left[\eta_{1}, \eta_{2}\right]$, and $\left|\mathcal{G}^{(2 \check{\alpha})}\right|^{\rho}$ is a generalized convex function on $\Omega$. Then

$$
\begin{aligned}
& \left|\left[\frac{\mathcal{G}\left(\eta_{1}\right)+\mathcal{G}\left(\eta_{2}\right)}{2^{\check{\alpha}}}\right] \Gamma(1+\check{\alpha})-\left(\frac{1}{\eta_{2}-\eta_{1}}\right)^{\check{\alpha}} \Gamma^{2}(1+\check{\alpha})_{\eta_{1}} \mathcal{I}_{\eta_{2}}^{(\check{\alpha})} \mathcal{G}(u)\right| \\
& \quad \leq \frac{\left(\eta_{2}-\eta_{1}\right)^{2 \check{\alpha}}}{16^{\check{\alpha}}}\left(\frac{1}{\Gamma(1+\check{\alpha})}-\frac{\Gamma(1+2 \check{\alpha})}{\Gamma(1+3 \check{\alpha})}\right)^{1-\frac{1}{\rho}}\left[\left[\Psi_{1}^{(\check{\alpha})}\left|\mathcal{G}^{(2 \check{\alpha})}\left(\eta_{1}\right)\right|^{\rho}+\Psi_{2}^{(\check{\alpha})}\left|\mathcal{G}^{(2 \check{\alpha})}\left(\eta_{2}\right)\right|^{\rho}\right]^{1 / \rho}\right. \\
& \left.\quad+\left[\Psi_{2}^{(\check{\alpha})}\left|\mathcal{G}^{(2 \check{\alpha})}\left(\eta_{1}\right)\right|^{\rho}+\Psi_{1}^{(\check{\alpha})}\left|\mathcal{G}^{(2 \check{\alpha})}\left(\eta_{2}\right)\right|^{\rho}\right]^{1 / \rho}\right]
\end{aligned}
$$

where

$$
\begin{aligned}
& \Psi_{1}^{(\check{\alpha})}=\frac{1}{2^{\check{\alpha}}}\left[\frac{1}{\Gamma(1+\check{\alpha})}-\frac{\Gamma(1+\check{\alpha})}{\Gamma(1+2 \check{\alpha})}-\frac{\Gamma(1+2 \check{\alpha})}{\Gamma(1+3 \check{\alpha})}+\frac{\Gamma(1+3 \check{\alpha})}{\Gamma(1+4 \check{\alpha})}\right], \\
& \Psi_{2}^{(\check{\alpha})}=\frac{1}{2^{\check{\alpha}}}\left[\frac{1}{\Gamma(1+\check{\alpha})}+\frac{\Gamma(1+\check{\alpha})}{\Gamma(1+2 \check{\alpha})}-\frac{\Gamma(1+2 \check{\alpha})}{\Gamma(1+3 \check{\alpha})}-\frac{\Gamma(1+3 \check{\alpha})}{\Gamma(1+4 \check{\alpha})}\right] .
\end{aligned}
$$

Proof It follows Lemma 5.1 and the generalized power-mean integral inequality that

$$
\begin{aligned}
& \left|\left[\frac{\mathcal{G}\left(\eta_{1}\right)+\mathcal{G}\left(\eta_{2}\right)}{2^{\check{\alpha}}}\right] \Gamma(1+\check{\alpha})-\left(\frac{1}{\eta_{2}-\eta_{1}}\right)^{\check{\alpha}} \Gamma^{2}(1+\check{\alpha})_{\eta_{1}} \mathcal{I}_{\eta_{2}}^{(\check{\alpha})} \mathcal{G}(u)\right| \\
& \leq \frac{\left(\eta_{2}-\eta_{1}\right)^{2 \check{\alpha}}}{16^{\check{\alpha}}}\left[\frac{1}{\Gamma(1+\check{\alpha})} \int_{0}^{1}\left(1-\vartheta^{2 \check{\alpha}}\right)\left|\mathcal{G}^{(\check{\alpha})}\left(\frac{1-\vartheta}{2} \eta_{1}+\frac{1+\vartheta}{2} \eta_{2}\right)\right|(d \vartheta)^{\check{\alpha}}\right. \\
& \left.\quad+\frac{1}{\Gamma(1+\check{\alpha})} \int_{0}^{1}\left(1-\vartheta^{2 \check{\alpha}}\right)\left|\mathcal{G}^{(2 \check{\alpha})}\left(\frac{1+\vartheta}{2} \eta_{1}+\frac{1-\vartheta}{2} \eta_{2}\right)\right|(d \vartheta)^{\check{\alpha}}\right] \\
& \leq \quad \frac{\left(\eta_{2}-\eta_{1}\right)^{2 \check{\alpha}}}{16^{\check{\alpha}}}\left[\left(\frac{1}{\Gamma(1+\check{\alpha})} \int_{0}^{1}\left(1-\vartheta^{2 \check{\alpha}}\right)(d \vartheta)^{\check{\alpha}}\right)^{1-\frac{1}{\rho}}\right. \\
& \quad \times\left(\frac{1}{\Gamma(1+\check{\alpha})} \int_{0}^{1}\left(1-\vartheta^{2 \check{\alpha}}\right)\left|\mathcal{G}^{(2 \check{\alpha})}\left(\frac{1-\vartheta}{2} \eta_{1}+\frac{1+\vartheta}{2} \eta_{2}\right)\right|^{\rho}(d \vartheta)^{\check{\alpha}}\right)^{1 / \rho} \\
& \quad+\left(\frac{1}{\Gamma(1+\check{\alpha})} \int_{0}^{1}\left(1-\vartheta^{2 \check{\alpha}}\right)(d \vartheta)^{\check{\alpha}}\right)^{1-\frac{1}{\rho}} \\
& \left.\quad \times\left(\frac{1}{\Gamma(1+\check{\alpha})} \int_{0}^{1}\left(1-\vartheta^{2 \check{\alpha}}\right)\left|\mathcal{G}^{(2 \check{\alpha})}\left(\frac{1+\vartheta}{2} \eta_{1}+\frac{1-\vartheta}{2} \eta_{2}\right)\right|^{\rho}(d \vartheta)^{\check{\alpha}}\right)^{1 / \rho}\right] .
\end{aligned}
$$


Since $\left|\mathcal{G}^{(2 \check{\alpha})}\right|^{\rho}$ is a generalized convex function on $\Omega$, we have

$$
\left|\mathcal{G}^{(2 \check{\alpha})}\left(\frac{1-\vartheta}{2} \eta_{1}+\frac{1+\vartheta}{2} \eta_{2}\right)\right|^{\rho} \leq\left(\frac{1-\vartheta}{2}\right)\left|\mathcal{G}^{(2 \check{\alpha})}\left(\eta_{1}\right)\right|^{\rho}+\left(\frac{1+\vartheta}{2}\right)^{\check{\alpha}}\left|\mathcal{G}^{(2 \check{\alpha})}\left(\eta_{2}\right)\right|^{\rho}
$$

and

$$
\left|\mathcal{G}^{(2 \check{\alpha})}\left(\frac{1+\vartheta}{2} \eta_{1}+\frac{1-\vartheta}{2} \eta_{2}\right)\right|^{\rho} \leq\left(\frac{1+\vartheta}{2}\right)^{\check{\alpha}}\left|\mathcal{G}^{(2 \check{\alpha})}\left(\eta_{1}\right)\right|^{\rho}+\left(\frac{1-\vartheta}{2}\right)^{\check{\alpha}}\left|\mathcal{G}^{(2 \check{\alpha})}\left(\eta_{2}\right)\right|^{\rho} .
$$

Therefore

$$
\begin{aligned}
\mid[ & \left.\frac{\mathcal{G}\left(\eta_{1}\right)+\mathcal{G}\left(\eta_{2}\right)}{2^{\check{\alpha}}}\right] \Gamma(1+\check{\alpha})-\left(\frac{1}{\eta_{2}-\eta_{1}}\right)^{\check{\alpha}} \Gamma^{2}(1+\check{\alpha})_{\eta_{1}} \mathcal{I}_{\eta_{2}}^{(\check{\alpha})} \mathcal{G}(u) \mid \\
\leq & \frac{\left(\eta_{2}-\eta_{1}\right)^{2 \check{\alpha}}}{16^{\check{\alpha}}}\left(\frac{1}{\Gamma(1+\check{\alpha})}-\frac{\Gamma(1+2 \check{\alpha})}{\Gamma(1+3 \check{\alpha})}\right)^{1-\frac{1}{\rho}}\left[\frac{1}{\Gamma(1+\check{\alpha})}\right. \\
& \times \int_{0}^{1}\left(1-\vartheta^{2 \check{\alpha}}\right)\left\{\left(\frac{1-\vartheta}{2}\right)^{\check{\alpha}}\left|\mathcal{G}^{(2 \check{\alpha})}\left(\eta_{1}\right)\right|^{\rho}+\left(\frac{1+\vartheta}{2}\right)^{\check{\alpha}}\left|\mathcal{G}^{(2 \check{\alpha})}\left(\eta_{2}\right)\right|^{\rho}\right\}(d \vartheta)^{\check{\alpha}} \\
& +\frac{1}{\Gamma(1+\check{\alpha})} \\
& \left.\times \int_{0}^{1}\left(1-\vartheta^{2 \check{\alpha}}\right)\left\{\left(\frac{1+\vartheta}{2}\right)^{\check{\alpha}}\left|\mathcal{G}^{(2 \check{\alpha})}\left(\eta_{1}\right)\right|^{\rho}+\left(\frac{1-\vartheta}{2}\right)^{\check{\alpha}}\left|\mathcal{G}^{(2 \check{\alpha})}\left(\eta_{2}\right)\right|^{\rho}\right\}(d \vartheta)^{\check{\alpha}}\right] \\
\leq & \frac{\left(\eta_{2}-\eta_{1}\right)^{2 \check{\alpha}}}{16^{\check{\alpha}}}\left(\frac{1}{\Gamma(1+\check{\alpha})}-\frac{\Gamma(1+2 \check{\alpha})}{\Gamma(1+3 \check{\alpha})}\right)^{1-\frac{1}{\rho}}\left[\left[\Psi_{1}^{(\check{\alpha})}\left|\mathcal{G}^{(2 \check{\alpha})}\left(\eta_{1}\right)\right|^{\rho}+\Psi_{2}^{(\check{\alpha})}\left|\mathcal{G}^{(2 \check{\alpha})}\left(\eta_{2}\right)\right|^{\rho}\right]^{1 / \rho}\right. \\
& \left.+\left[\Psi_{2}^{(\check{\alpha})}\left|\mathcal{G}^{(2 \check{\alpha})}\left(\eta_{1}\right)\right|^{\rho}+\Psi_{1}^{(\check{\alpha})}\left|\mathcal{G}^{(2 \check{\alpha})}\left(\eta_{2}\right)\right|^{\rho}\right]^{1 / \rho}\right],
\end{aligned}
$$

where we have used the equalities

$$
\begin{aligned}
\Psi_{1}^{(\check{\alpha})} & :=\frac{1}{\Gamma(1+\check{\alpha})} \int_{0}^{1}\left(1-\vartheta^{2 \check{\alpha}}\right)(1-\vartheta)^{\check{\alpha}}(d \vartheta)^{\check{\alpha}} \\
& =\frac{1}{2^{\check{\alpha}}}\left[\frac{1}{\Gamma(1+\check{\alpha})}-\frac{\Gamma(1+\check{\alpha})}{\Gamma(1+2 \check{\alpha})}-\frac{\Gamma(1+2 \check{\alpha})}{\Gamma(1+3 \check{\alpha})}+\frac{\Gamma(1+3 \check{\alpha})}{\Gamma(1+4 \check{\alpha})}\right], \\
\Psi_{2}^{(\check{\alpha})} & :=\frac{1}{\Gamma(1+\check{\alpha})} \int_{0}^{1}\left(1-\vartheta^{2 \check{\alpha}}\right)(1+\vartheta)^{\check{\alpha}}(d \vartheta)^{\check{\alpha}} \\
& =\frac{1}{2^{\check{\alpha}}}\left[\frac{1}{\Gamma(1+\check{\alpha})}+\frac{\Gamma(1+\check{\alpha})}{\Gamma(1+2 \check{\alpha})}-\frac{\Gamma(1+2 \check{\alpha})}{\Gamma(1+3 \check{\alpha})}-\frac{\Gamma(1+3 \check{\alpha})}{\Gamma(1+4 \check{\alpha})}\right] .
\end{aligned}
$$

This completes the proof.

\section{Generalized integral inequalities for third-order local differentiable functions}

In this section, we present some novel variants of Hermite-Hadamard-type inequality for the functions with generalized convex third local fractional derivatives. For this purpose, we need the following identity. 
Lemma 6.1 Let $\check{\alpha} \in(0,1]$, and let a mapping $\mathcal{G}: \Omega \rightarrow \mathbb{R}^{\check{\alpha}}$ be such that $\mathcal{G} \in \mathcal{D}_{\check{\alpha}}(\Omega)$ and $\mathcal{G}^{(3 \check{\alpha})} \in \mathcal{C}_{\check{\alpha}}\left[\eta_{1}, \eta_{2}\right]$. Then

$$
\begin{aligned}
\Gamma^{2}(1 & +\check{\alpha}) \mathcal{G}\left(\frac{\eta_{1}+\eta_{2}}{2}\right)+\left(\frac{\eta_{2}-\eta_{1}}{24}\right)^{\check{\alpha}} \Gamma(1+\check{\alpha})\left[\mathcal{G}^{(\check{\alpha})}\left(\eta_{2}\right)-\mathcal{G}^{(\check{\alpha})}\left(\eta_{1}\right)\right] \\
& -\left(\frac{1}{\eta_{2}-\eta_{1}}\right)^{\check{\alpha}} \Gamma^{3}(1+\check{\alpha})_{\eta_{1}} \mathcal{I}_{\eta_{2}(\check{\alpha})} \\
= & \left(\frac{\left(\eta_{2}-\eta_{1}\right)^{3}}{96}\right)^{\check{\alpha}}\left[\frac{1}{\Gamma(1+\check{\alpha})}\right. \\
& \times \int_{0}^{1} \vartheta^{\check{\alpha}}(1-\vartheta)^{\check{\alpha}}(2-\vartheta)^{\check{\alpha}} \mathcal{G}^{(3 \check{\alpha})}\left(\frac{1-\vartheta}{2} \eta_{1}+\frac{1+\vartheta}{2} \eta_{2}\right)(d \vartheta)^{\check{\alpha}} \\
& \left.-\frac{1}{\Gamma(1+\check{\alpha})} \int_{0}^{1} \vartheta^{\check{\alpha}}(1-\vartheta)^{\check{\alpha}}(2-\vartheta)^{\check{\alpha}} \mathcal{G}^{(3 \check{\alpha})}\left(\frac{1+\vartheta}{2} \eta_{1}+\frac{1-\vartheta}{2} \eta_{2}\right)(d \vartheta)^{\check{\alpha}}\right] .
\end{aligned}
$$

Proof Using the local fractional integration by parts and change of variable leads to

$$
\begin{aligned}
& \frac{1}{\Gamma(1+\check{\alpha})} \int_{0}^{1} \vartheta^{\check{\alpha}}(1-\vartheta)^{\check{\alpha}}(2-\vartheta)^{\check{\alpha}} \mathcal{G}^{(3 \check{\alpha})}\left(\frac{1-\vartheta}{2} \eta_{1}+\frac{1+\vartheta}{2} \eta_{2}\right)(d \vartheta)^{\check{\alpha}} \\
& =\left.\left(\frac{2}{\eta_{2}-\eta_{1}}\right)^{\check{\alpha}} \vartheta^{\check{\alpha}}(1-\vartheta)^{\check{\alpha}}(2-\vartheta)^{\check{\alpha}} \mathcal{G}^{(2 \check{\alpha})}\left(\frac{1-\vartheta}{2} \eta_{1}+\frac{1+\vartheta}{2} \eta_{2}\right)\right|_{0} ^{1} \\
& -\left(\frac{2}{\eta_{2}-\eta_{1}}\right)^{\check{\alpha}} \frac{\Gamma(1+\check{\alpha})}{\Gamma(1+\check{\alpha})} \\
& \times \int_{0}^{1}\left(3^{\check{\alpha}} \vartheta^{2 \check{\alpha}}-(6 \vartheta)^{\check{\alpha}}+2^{\check{\alpha}}\right) \mathcal{G}^{(2 \check{\alpha})}\left(\frac{1-\vartheta}{2} \eta_{1}+\frac{1+\vartheta}{2} \eta_{2}\right)(d \vartheta)^{\check{\alpha}} \\
& =\Gamma(1+\check{\alpha})\left(\frac{4}{\left(\eta_{2}-\eta_{1}\right)^{2}}\right)^{\check{\alpha}}\left[\mathcal{G}^{(\check{\alpha})}\left(\eta_{2}\right)+2^{\check{\alpha}} \mathcal{G}^{(\check{\alpha})}\left(\frac{\eta_{1}+\eta_{2}}{2}\right)\right] \\
& -\left(\frac{24}{\left(\eta_{2}-\eta_{1}\right)^{2}}\right)^{\check{\alpha}} \frac{\Gamma^{2}(1+\check{\alpha})}{\Gamma(1+\check{\alpha})} \int_{0}^{1}(1-\vartheta)^{\check{\alpha}} \mathcal{G}^{(\check{\alpha})}\left(\frac{1-\vartheta}{2} \eta_{1}+\frac{1+\vartheta}{2} \eta_{2}\right)(d \vartheta)^{\check{\alpha}} \\
& =\Gamma(1+\check{\alpha})\left(\frac{4}{\left(\eta_{2}-\eta_{1}\right)^{2}}\right)^{\check{\alpha}}\left[\mathcal{G}^{(\check{\alpha})}\left(\eta_{2}\right)+2^{\check{\alpha}} \mathcal{G}^{(\check{\alpha})}\left(\frac{\eta_{1}+\eta_{2}}{2}\right)\right] \\
& +\left(\frac{48}{\left(\eta_{2}-\eta_{1}\right)^{3}}\right)^{\check{\alpha}} \Gamma^{2}(1+\check{\alpha}) \mathcal{G}\left(\frac{\eta_{1}+\eta_{2}}{2}\right) \\
& -\left(\frac{48}{\left(\eta_{2}-\eta_{1}\right)^{3}}\right)^{\check{\alpha}} \frac{\Gamma^{3}(1+\check{\alpha})}{\Gamma(1+\check{\alpha})} \int_{0}^{1} \mathcal{G}\left(\frac{1-\vartheta}{2} \eta_{1}+\frac{1+\vartheta}{2} \eta_{2}\right)(d \vartheta)^{\check{\alpha}} \\
& =\Gamma(1+\check{\alpha})\left(\frac{4}{\left(\eta_{2}-\eta_{1}\right)^{2}}\right)^{\check{\alpha}}\left[\mathcal{G}^{(\check{\alpha})}\left(\eta_{2}\right)+2^{\check{\alpha}} \mathcal{G}^{(\check{\alpha})}\left(\frac{\eta_{1}+\eta_{2}}{2}\right)\right] \\
& +\left(\frac{48}{\left(\eta_{2}-\eta_{1}\right)^{3}}\right)^{\check{\alpha}} \Gamma^{2}(1+\check{\alpha}) \mathcal{G}\left(\frac{\eta_{1}+\eta_{2}}{2}\right) \\
& -\left(\frac{96}{\left(\eta_{2}-\eta_{1}\right)^{4}}\right)^{\check{\alpha}} \Gamma^{3}(1+\check{\alpha})_{\frac{\eta_{1}+\eta_{2}}{2}} \mathcal{I}_{\eta_{2}}^{(\check{\alpha})} \mathcal{G}(u) .
\end{aligned}
$$


Analogously, we have

$$
\begin{aligned}
& \frac{1}{\Gamma(1+\check{\alpha})} \int_{0}^{1} \vartheta^{\check{\alpha}}(1-\vartheta)^{\check{\alpha}}(2-\vartheta)^{\check{\alpha}} \mathcal{G}^{(3 \check{\alpha})}\left(\frac{1+\vartheta}{2} \eta_{1}+\frac{1-\vartheta}{2} \eta_{2}\right)(d \vartheta)^{\check{\alpha}} \\
& =\Gamma(1+\check{\alpha})\left(\frac{4}{\left(\eta_{2}-\eta_{1}\right)^{2}}\right)^{\check{\alpha}}\left[\mathcal{G}^{(\check{\alpha})}\left(\eta_{1}\right)+2^{\check{\alpha}} \mathcal{G}^{(\check{\alpha})}\left(\frac{\eta_{1}+\eta_{2}}{2}\right)\right] \\
& \quad-\left(\frac{48}{\left(\eta_{2}-\eta_{1}\right)^{3}}\right)^{\check{\alpha}} \Gamma^{2}(1+\check{\alpha}) \mathcal{G}\left(\frac{\eta_{1}+\eta_{2}}{2}\right) \\
& +\left(\frac{96}{\left(\eta_{2}-\eta_{1}\right)^{4}}\right)^{\check{\alpha}} \Gamma^{3}(1+\check{\alpha})_{\eta_{1}} \mathcal{I}_{\frac{\eta_{1}+\eta_{2}}{2}}^{(\check{\alpha})} \mathcal{G}(u) .
\end{aligned}
$$

Subtracting the above identities side by side and then multiplying the obtained identity by $\left(\frac{\left(\eta_{2}-\eta_{1}\right)^{3}}{96}\right)^{\check{\alpha}}$, we get the desired result.

Theorem 6.2 Let $\rho, \omega>1$ with $\rho^{-1}+\omega^{-1}=1$, and let a mapping $\mathcal{G}: \Omega \rightarrow \mathbb{R}^{\check{\alpha}}(0<\check{\alpha} \leq 1)$ be such that $\mathcal{G} \in \mathcal{D}_{\check{\alpha}}(\Omega), \mathcal{G}^{(3 \check{\alpha})} \in \mathcal{C}_{\check{\alpha}}\left[\eta_{1}, \eta_{2}\right]$, and $\left|\mathcal{G}^{(3 \check{\alpha})}\right|^{\rho}$ is a generalized convex function on

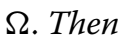

$$
\begin{aligned}
\mid \Gamma^{2}(1+\check{\alpha}) \mathcal{G}\left(\frac{\eta_{1}+\eta_{2}}{2}\right)+\left(\frac{\eta_{2}-\eta_{1}}{24}\right)^{\check{\alpha}} \Gamma(1+\check{\alpha})\left[\mathcal{G}^{(\check{\alpha})}\left(\eta_{2}\right)-\mathcal{G}^{(\check{\alpha})}\left(\eta_{1}\right)\right] \\
-\left(\frac{1}{\eta_{2}-\eta_{1}}\right)^{\check{\alpha}} \Gamma^{3}(1+\check{\alpha})_{\eta_{1}} \mathcal{I}_{\eta_{2}}^{(\check{\alpha})} \mid \\
\leq\left(\frac{\left(\eta_{2}-\eta_{1}\right)^{3}}{96}\right)^{\check{\alpha}}\left(\Psi_{1}^{*}\right)^{1-1 / \rho}\left[\left[\left(\Psi_{2}^{*}\right)\left|\mathcal{G}^{(3 \check{\alpha})}\left(\eta_{1}\right)\right|^{\rho}+\left(\Psi_{3}^{*}\right)\left|\mathcal{G}^{(3 \check{\alpha})}\left(\eta_{2}\right)\right|^{\rho}\right]^{1 / \rho}\right. \\
\left.+\left[\left(\Psi_{3}^{*}\right)\left|\mathcal{G}^{(3 \check{\alpha})}\left(\eta_{1}\right)\right|^{\rho}+\left(\Psi_{2}^{*}\right)\left|\mathcal{G}^{(3 \check{\alpha})}\left(\eta_{2}\right)\right|^{\rho}\right]^{1 / \rho}\right],
\end{aligned}
$$

where

$$
\begin{aligned}
& \Psi_{1}^{*}=\frac{\Gamma(1+3 \check{\alpha})}{\Gamma(1+4 \check{\alpha})}-3^{\check{\alpha}} \frac{\Gamma(1+2 \check{\alpha})}{\Gamma(1+3 \check{\alpha})}+2^{\check{\alpha}} \frac{\Gamma(1+\check{\alpha})}{\Gamma(1+2 \check{\alpha})} \\
& \Psi_{2}^{*}=\left(\frac{1}{2}\right)^{\check{\alpha}}\left[2^{\check{\alpha}} \frac{\Gamma(1+\check{\alpha})}{\Gamma(1+2 \check{\alpha})}-5^{\check{\alpha}} \frac{\Gamma(1+2 \check{\alpha})}{\Gamma(1+3 \check{\alpha})}+4^{\check{\alpha}} \frac{\Gamma(1+3 \check{\alpha})}{\Gamma(1+4 \check{\alpha})}-\frac{\Gamma(1+4 \check{\alpha})}{\Gamma(1+5 \check{\alpha})}\right],
\end{aligned}
$$

and

$$
\Psi_{3}^{*}:=\left(\frac{1}{2}\right)^{\check{\alpha}}\left[2^{\check{\alpha}}\left[\frac{\Gamma(1+\check{\alpha})}{\Gamma(1+2 \check{\alpha})}-\frac{\Gamma(1+3 \check{\alpha})}{\Gamma(1+4 \check{\alpha})}\right]+\frac{\Gamma(1+4 \check{\alpha})}{\Gamma(1+5 \check{\alpha})}-\frac{\Gamma(1+2 \check{\alpha})}{\Gamma(1+3 \check{\alpha})}\right] .
$$

Proof From Lemma 6.1 and the generalized Hölder integral inequality we get

$$
\begin{aligned}
\mid & \Gamma^{2}(1+\check{\alpha}) \mathcal{G}\left(\frac{\eta_{1}+\eta_{2}}{2}\right)+\left(\frac{\eta_{2}-\eta_{1}}{24}\right)^{\check{\alpha}} \Gamma(1+\check{\alpha})\left[\mathcal{G}^{(\check{\alpha})}\left(\eta_{2}\right)-\mathcal{G}^{(\check{\alpha})}\left(\eta_{1}\right)\right] \\
& -\left(\frac{1}{\eta_{2}-\eta_{1}}\right)^{\check{\alpha}} \Gamma^{3}(1+\check{\alpha})_{\eta_{1}} \mathcal{I}_{\eta_{2}}^{(\check{\alpha})} \mid \\
\leq & \left(\frac{\left(\eta_{2}-\eta_{1}\right)^{3}}{96}\right)^{\check{\alpha}}\left[\frac{1}{\Gamma(1+\check{\alpha})}\right.
\end{aligned}
$$


Khan et al. Advances in Difference Equations

(2020) 2020:657

Page 17 of 24

$$
\begin{aligned}
& \times \int_{0}^{1} \vartheta^{\check{\alpha}}(1-\vartheta)^{\check{\alpha}}(2-\vartheta)^{\check{\alpha}}\left|\mathcal{G}^{(3 \check{\alpha})}\left(\frac{1-\vartheta}{2} \eta_{1}+\frac{1+\vartheta}{2} \eta_{2}\right)\right|(d \vartheta)^{\check{\alpha}} \\
& \left.+\frac{1}{\Gamma(1+\check{\alpha})} \int_{0}^{1} \vartheta^{\check{\alpha}}(1-\vartheta)^{\check{\alpha}}(2-\vartheta)^{\check{\alpha}}\left|\mathcal{G}^{(3 \check{\alpha})}\left(\frac{1+\vartheta}{2} \eta_{1}+\frac{1-\vartheta}{2} \eta_{2}\right)\right|(d \vartheta)^{\check{\alpha}}\right] \\
& \leq\left(\frac{\left(\eta_{2}-\eta_{1}\right)^{3}}{96}\right)^{\check{\alpha}}\left[\left(\frac{1}{\Gamma(1+\check{\alpha})} \int_{0}^{1} \vartheta^{\check{\alpha}}(1-\vartheta)^{\check{\alpha}}(2-\vartheta)^{\check{\alpha}}(d \vartheta)^{\check{\alpha}}\right)^{1-1 / \rho}\right. \\
& \times\left(\frac{1}{\Gamma(1+\check{\alpha})} \int_{0}^{1} \vartheta^{\check{\alpha}}(1-\vartheta)^{\check{\alpha}}(2-\vartheta)^{\check{\alpha}}\left|\mathcal{G}^{(3 \check{\alpha})}\left(\frac{1-\vartheta}{2} \eta_{1}+\frac{1+\vartheta}{2} \eta_{2}\right)\right|^{\rho}(d \vartheta)^{\check{\alpha}}\right)^{1 / \rho} \\
& +\left(\frac{1}{\Gamma(1+\check{\alpha})} \int_{0}^{1} \vartheta^{\check{\alpha}}(1-\vartheta)^{\check{\alpha}}(2-\vartheta)^{\check{\alpha}}(d \vartheta)^{\check{\alpha}}\right)^{1-1 / \rho} \\
& \times\left(\frac{1}{\Gamma(1+\check{\alpha})} \int_{0}^{1} \vartheta^{\check{\alpha}}(1-\vartheta)^{\check{\alpha}}(2-\vartheta)^{\check{\alpha}}\right. \\
& \left.\left.\times\left|\mathcal{G}^{(3 \check{\alpha})}\left(\frac{1+\vartheta}{2} \eta_{1}+\frac{1-\vartheta}{2} \eta_{2}\right)\right|^{\rho}(d \vartheta)^{\check{\alpha}}\right)^{1 / \rho}\right] .
\end{aligned}
$$

Since $\left|\mathcal{G}^{(3 \check{\alpha})}\right|^{\rho}$ is a generalized convex function on $\Omega$, we have

$$
\left|\mathcal{G}^{(3 \check{\alpha})}\left(\frac{1+\vartheta}{2} \eta_{1}+\frac{1-\vartheta}{2} \eta_{2}\right)\right|^{\rho} \leq\left(\frac{1+\vartheta}{2}\right)^{\check{\alpha}}\left|\mathcal{G}^{(3 \check{\alpha})}\left(\eta_{1}\right)\right|^{\rho}+\left(\frac{1-\vartheta}{2}\right)^{\check{\alpha}}\left|\mathcal{G}^{(3 \check{\alpha})}\left(\eta_{2}\right)\right|^{\rho}
$$

and

$$
\left|\mathcal{G}^{(3 \check{\alpha})}\left(\frac{1-\vartheta}{2} \eta_{1}+\frac{1+\vartheta}{2} \eta_{2}\right)\right|^{\rho} \leq\left(\frac{1-\vartheta}{2}\right)^{\check{\alpha}}\left|\mathcal{G}^{(3 \check{\alpha})}\left(\eta_{1}\right)\right|^{\rho}+\left(\frac{1+\vartheta}{2}\right)^{\check{\alpha}}\left|\mathcal{G}^{(3 \check{\alpha})}\left(\eta_{2}\right)\right|^{\rho} .
$$

It follows from (6.7) that

$$
\begin{aligned}
& \mid \mathcal{G}\left(\frac{\eta_{1}+\eta_{2}}{2}\right)-\left(\frac{1}{\eta_{2}-\eta_{1}}\right)^{\check{\alpha}} \Gamma(1+\check{\alpha})_{\eta_{1}} \mathcal{I}_{\eta_{2}}^{(\check{\alpha})} \\
& \quad+\left(\frac{\eta_{2}-\eta_{1}}{24}\right)^{\check{\alpha}} \frac{1}{\Gamma(1+\check{\alpha})}\left[\mathcal{G}^{(\check{\alpha})}\left(\eta_{2}\right)-\mathcal{G}^{(\check{\alpha})}\left(\eta_{1}\right)\right] \mid \\
& \leq\left(\frac{\left(\eta_{2}-\eta_{1}\right)^{3}}{96}\right)^{\check{\alpha}}\left[\left(\frac{1}{\Gamma(1+\check{\alpha})} \int_{0}^{1} \vartheta^{\check{\alpha}}(1-\vartheta)^{\check{\alpha}}(2-\vartheta)^{\check{\alpha}}(d \vartheta)^{\check{\alpha}}\right)^{1-1 / \rho}\right. \\
& \times\left(\frac{1}{\Gamma(1+\check{\alpha})} \int_{0}^{1} \vartheta^{\check{\alpha}}(1-\vartheta)^{\check{\alpha}}(2-\vartheta)^{\check{\alpha}}\right. \\
&\left.\times\left[\left(\frac{1+\vartheta}{2}\right)^{\check{\alpha}}\left|\mathcal{G}^{(3 \check{\alpha})}\left(\eta_{1}\right)\right|^{\rho}+\left(\frac{1-\vartheta}{2}\right)^{\check{\alpha}}\left|\mathcal{G}^{(3 \check{\alpha})}\left(\eta_{2}\right)\right|^{\rho}\right](d \vartheta)^{\check{\alpha}}\right)^{1 / \rho} \\
&+\left(\frac{1}{\Gamma(1+\check{\alpha})} \int_{0}^{1} \vartheta^{\check{\alpha}}(1-\vartheta)^{\check{\alpha}}(2-\vartheta)^{\check{\alpha}}(d \vartheta)^{\check{\alpha}}\right)^{1-1 / \rho} \\
& \times\left(\frac{1}{\Gamma(1+\check{\alpha})} \int_{0}^{1} \vartheta^{\check{\alpha}}(1-\vartheta)^{\check{\alpha}}(2-\vartheta)^{\check{\alpha}}\right. \\
&\left.\left.\times\left[\left(\frac{1-\vartheta}{2}\right)^{\check{\alpha}}\left|\mathcal{G}^{(3 \check{\alpha})}\left(\eta_{1}\right)^{\rho}+\left(\frac{1+\vartheta}{2}\right)^{\check{\alpha}}\right| \mathcal{G}^{(3 \check{\alpha})}\left(\eta_{2}\right)^{\rho}\right](d \vartheta)^{\check{\alpha}}\right)^{1 / \rho}\right]
\end{aligned}
$$




$$
\begin{aligned}
\leq & \left(\frac{\left(\eta_{2}-\eta_{1}\right)^{3}}{96}\right)^{\check{\alpha}}\left(\Psi_{1}^{*}\right)^{1-1 / \rho}\left[\left[\left(\Psi_{2}^{*}\right)\left|\mathcal{G}^{(3 \check{\alpha})}\left(\eta_{1}\right)\right|^{\rho}+\left(\Psi_{3}^{*}\right)\left|\mathcal{G}^{(3 \check{\alpha})}\left(\eta_{2}\right)\right|^{\rho}\right]^{1 / \rho}\right. \\
& \left.+\left[\left(\Psi_{3}^{*}\right)\left|\mathcal{G}^{(3 \check{\alpha})}\left(\eta_{1}\right)\right|^{\rho}+\left(\Psi_{2}^{*}\right)\left|\mathcal{G}^{(3 \check{\alpha})}\left(\eta_{2}\right)\right|^{\rho}\right]^{1 / \rho}\right],
\end{aligned}
$$

where we have used the equalities

$$
\begin{aligned}
\Psi_{1}^{*} & :=\frac{1}{\Gamma(1+\check{\alpha})} \int_{0}^{1} \vartheta^{\check{\alpha}}(1-\vartheta)^{\check{\alpha}}(2-\vartheta)^{\check{\alpha}}(d \vartheta)^{\check{\alpha}} \\
& =\frac{\Gamma(1+3 \check{\alpha})}{\Gamma(1+4 \check{\alpha})}-3^{\check{\alpha}} \frac{\Gamma(1+2 \check{\alpha})}{\Gamma(1+3 \check{\alpha})}+2^{\check{\alpha}} \frac{\Gamma(1+\check{\alpha})}{\Gamma(1+2 \check{\alpha})}, \\
\Psi_{2}^{*} & :=\left(\frac{1}{2}\right)^{\check{\alpha}} \frac{1}{\Gamma(1+\check{\alpha})} \int_{0}^{1} \vartheta^{\check{\alpha}}(1-\vartheta)^{2 \check{\alpha}}(2-\vartheta)^{\check{\alpha}}(d \vartheta)^{\check{\alpha}} \\
& =\left(\frac{1}{2}\right)^{\check{\alpha}}\left[2^{\check{\alpha}} \frac{\Gamma(1+\check{\alpha})}{\Gamma(1+2 \check{\alpha})}-5^{\check{\alpha}} \frac{\Gamma(1+2 \check{\alpha})}{\Gamma(1+3 \check{\alpha})}+4^{\check{\alpha}} \frac{\Gamma(1+3 \check{\alpha})}{\Gamma(1+4 \check{\alpha})}-\frac{\Gamma(1+4 \check{\alpha})}{\Gamma(1+5 \check{\alpha})}\right], \\
\Psi_{3}^{*} & :=\left(\frac{1}{2}\right)^{\check{\alpha}} \frac{1}{\Gamma(1+\check{\alpha})} \int_{0}^{1} \vartheta^{\check{\alpha}}(1+\vartheta)^{\check{\alpha}}(1-\vartheta)^{\check{\alpha}}(2-\vartheta)^{\check{\alpha}}(d \vartheta)^{\check{\alpha}} \\
& =\left(\frac{1}{2}\right)^{\check{\alpha}}\left[2^{\check{\alpha}}\left[\frac{\Gamma(1+\check{\alpha})}{\Gamma(1+2 \check{\alpha})}-\frac{\Gamma(1+3 \check{\alpha})}{\Gamma(1+4 \check{\alpha})}\right]+\frac{\Gamma(1+4 \check{\alpha})}{\Gamma(1+5 \check{\alpha})}-\frac{\Gamma(1+2 \check{\alpha})}{\Gamma(1+3 \check{\alpha})}\right] .
\end{aligned}
$$

This completes the proof of Theorem 6.2.

Corollary 6.3 If we choose $\rho=1$, then under the assumptions of Theorem 6.2, we get

$$
\begin{aligned}
\mid & \Gamma^{2}(1+\check{\alpha}) \mathcal{G}\left(\frac{\eta_{1}+\eta_{2}}{2}\right)+\left(\frac{\eta_{2}-\eta_{1}}{24}\right)^{\check{\alpha}} \Gamma(1+\check{\alpha})\left[\mathcal{G}^{(\check{\alpha})}\left(\eta_{2}\right)-\mathcal{G}^{(\check{\alpha})}\left(\eta_{1}\right)\right] \\
& -\left(\frac{1}{\eta_{2}-\eta_{1}}\right)^{\check{\alpha}} \Gamma^{3}(1+\check{\alpha})_{\eta_{1}} \mathcal{I}_{\eta_{2}}^{(\check{\alpha})} \mid \\
\leq & \left(\frac{\left(\eta_{2}-\eta_{1}\right)^{3}}{48}\right)^{\check{\alpha}}\left[\left(\Psi_{2}^{*}\right)\left|\mathcal{G}^{(3 \check{\alpha})}\left(\eta_{1}\right)\right|+\left(\Psi_{3}^{*}\right)\left|\mathcal{G}^{(3 \check{\alpha})}\left(\eta_{2}\right)\right|\right],
\end{aligned}
$$

where $\Psi_{2}^{*}$ and $\Psi_{3}^{*}$ are given in (6.5) and (6.6), respectively.

Remark 6.4 Letting $\check{\alpha}=1$, Theorem 6.2 reduces to Theorem 3.1 of [26].

Theorem 6.5 Let $\rho, \omega>1$ with $\rho^{-1}+\omega^{-1}=1$, and let a mapping $\mathcal{G}: \Omega \rightarrow \mathbb{R}^{\check{\alpha}}(0<\check{\alpha} \leq 1)$ be such that $\mathcal{G} \in \mathcal{D}_{\check{\alpha}}(\Omega), \mathcal{G}^{(3 \check{\alpha})} \in \mathcal{C}_{\check{\alpha}}\left[\eta_{1}, \eta_{2}\right]$, and $\left|\mathcal{G}^{(3 \check{)})}\right|^{\rho}$ is a generalized convex function on $\Omega$. Then

$$
\begin{aligned}
\mid \Gamma^{2}(1 & +\check{\alpha}) \mathcal{G}\left(\frac{\eta_{1}+\eta_{2}}{2}\right)+\left(\frac{\eta_{2}-\eta_{1}}{24}\right)^{\check{\alpha}} \Gamma(1+\check{\alpha})\left[\mathcal{G}^{(\check{\alpha})}\left(\eta_{2}\right)-\mathcal{G}^{(\check{\alpha})}\left(\eta_{1}\right)\right] \\
& -\left(\frac{1}{\eta_{2}-\eta_{1}}\right)^{\check{\alpha}} \Gamma^{3}(1+\check{\alpha})_{\eta_{1}} \mathcal{I}_{\eta_{2}}^{(\check{\alpha})} \mid \\
\leq & \left(\frac{\left(\eta_{2}-\eta_{1}\right)^{3}}{96}\right)^{\check{\alpha}}\left(\Psi_{1}^{* * *}\right)^{1-1 / \rho}\left[\left[\left(\Psi_{2}^{* * *}\right)\left|\mathcal{G}^{(3 \check{\alpha})}\left(\eta_{1}\right)\right|^{\rho}+\left(\Psi_{3}^{* * *}\right)\left|\mathcal{G}^{(3 \check{\alpha})}\left(\eta_{2}\right)\right|^{\rho}\right]^{1 / \rho}\right. \\
& \left.+\left[\left(\Psi_{3}^{* * *}\right)\left|\mathcal{G}^{(3 \check{\alpha})}\left(\eta_{1}\right)\right|^{\rho}+\left(\Psi_{2}^{* * *}\right)\left|\mathcal{G}^{(3 \check{\alpha})}\left(\eta_{2}\right)\right|^{\rho}\right]^{1 / \rho}\right],
\end{aligned}
$$


where

$$
\begin{aligned}
\Psi_{1}^{* * *}= & {\left[\frac{\Gamma(1+\check{\alpha})}{\Gamma(1+2 \check{\alpha})}-\frac{\Gamma(1+2 \check{\alpha})}{\Gamma(1+3 \check{\alpha})}\right]+\frac{\Gamma\left(1+\check{\alpha}\left(\frac{2 \rho-m-2}{\rho-1}\right)\right)}{\Gamma\left(1+\check{\alpha}\left(\frac{3 \rho-m-2}{\rho-1}\right)\right)}-\frac{\Gamma\left(1+\check{\alpha}\left(\frac{3 \rho-m-2}{\rho-1}\right)\right)}{\Gamma\left(1+\check{\alpha}\left(\frac{4 \rho-m-3}{\rho-1}\right)\right)}, } \\
\Psi_{2}^{* * *}= & {\left[\frac{\Gamma(1+\check{\alpha})}{\Gamma(1+2 \check{\alpha})}+\frac{\Gamma(1+(m+1) \check{\alpha})}{\Gamma(1+(m+2) \check{\alpha})}-\frac{\Gamma(1+2 \check{\alpha})}{\Gamma(1+3 \check{\alpha})}-\frac{\Gamma(1+(m+2) \check{\alpha})}{\Gamma(1+(m+3) \check{\alpha})}\right] } \\
& -\left(\frac{1}{2}\right)^{\check{\alpha}}\left[\frac{\Gamma(1+2 \check{\alpha})}{\Gamma(1+3 \check{\alpha})}-\frac{\Gamma(1+3 \check{\alpha})}{\Gamma(1+4 \check{\alpha})}+\frac{\Gamma(1+(m+2) \check{\alpha})}{\Gamma(1+(m+3) \check{\alpha})}-\frac{\Gamma(1+(m+3) \check{\alpha})}{\Gamma(1+(m+4) \check{\alpha})}\right], \\
\Psi_{3}^{* * *}= & \left(\frac{1}{2}\right)^{\check{\alpha}}\left[\frac{\Gamma(1+2 \check{\alpha})}{\Gamma(1+3 \check{\alpha})}-\frac{\Gamma(1+3 \check{\alpha})}{\Gamma(1+4 \check{\alpha})}+\frac{\Gamma(1+(m+2) \check{\alpha})}{\Gamma(1+(m+3) \check{\alpha})}-\frac{\Gamma(1+(m+3) \check{\alpha})}{\Gamma(1+(m+4) \check{\alpha})}\right] .
\end{aligned}
$$

Proof Using Lemma 6.1 and the generalized Hölder integral inequality, we have

$$
\begin{aligned}
& \mid \Gamma^{2}(1+\check{\alpha}) \mathcal{G}\left(\frac{\eta_{1}+\eta_{2}}{2}\right)+\left(\frac{\eta_{2}-\eta_{1}}{24}\right)^{\check{\alpha}} \Gamma(1+\check{\alpha})\left[\mathcal{G}^{(\check{\alpha})}\left(\eta_{2}\right)-\mathcal{G}^{(\check{\alpha})}\left(\eta_{1}\right)\right] \\
& -\left(\frac{1}{\eta_{2}-\eta_{1}}\right)^{\check{\alpha}} \Gamma^{3}(1+\check{\alpha})_{\eta_{1}} \mathcal{I}_{\eta_{2}}^{(\check{\alpha}} \\
& \leq\left(\frac{\left(\eta_{2}-\eta_{1}\right)^{3}}{96}\right)^{\check{\alpha}}\left[\frac{1}{\Gamma(1+\check{\alpha})}\right. \\
& \times \int_{0}^{1} \vartheta^{\check{\alpha}}(1-\vartheta)^{\check{\alpha}}(2-\vartheta)^{\check{\alpha}}\left|\mathcal{G}^{(3 \check{\alpha})}\left(\frac{1-\vartheta}{2} \eta_{1}+\frac{1+\vartheta}{2} \eta_{2}\right)\right|(d \vartheta)^{\check{\alpha}} \\
& \left.+\frac{1}{\Gamma(1+\check{\alpha})} \int_{0}^{1} \vartheta^{\check{\alpha}}(1-\vartheta)^{\check{\alpha}}(2-\vartheta)^{\check{\alpha}}\left|\mathcal{G}^{(3 \check{\alpha})}\left(\frac{1+\vartheta}{2} \eta_{1}+\frac{1-\vartheta}{2} \eta_{2}\right)\right|(d \vartheta)^{\check{\alpha}}\right] \\
& \leq\left(\frac{\left(\eta_{2}-\eta_{1}\right)^{3}}{96}\right)^{\check{\alpha}}\left[\left(\frac{1}{\Gamma(1+\check{\alpha})} \int_{0}^{1} \vartheta^{\check{\alpha}}(1-\vartheta)^{\check{\alpha}}(2-\vartheta)^{\check{\alpha}\left(\frac{\rho-m}{\rho-1}\right)}(d \vartheta)^{\check{\alpha}}\right)^{1-1 / \rho}\right. \\
& \times\left(\frac{1}{\Gamma(1+\check{\alpha})} \int_{0}^{1} \vartheta^{\check{\alpha}}(1-\vartheta)^{\check{\alpha}}(2-\vartheta)^{m \check{\alpha}}\left|\mathcal{G}^{(\check{\alpha})}\left(\frac{1-\vartheta}{2} \eta_{1}+\frac{1+\vartheta}{2} \eta_{2}\right)\right|^{\rho}(d \vartheta)^{\check{\alpha}}\right)^{1 / \rho} \\
& +\left(\frac{1}{\Gamma(1+\check{\alpha})} \int_{0}^{1} \vartheta^{\check{\alpha}}(1-\vartheta)^{\check{\alpha}}(2-\vartheta)^{\check{\alpha}\left(\frac{\rho-m}{\rho-1}\right)}(d \vartheta)^{\check{\alpha}}\right)^{1-1 / \rho} \\
& \left.\times\left(\frac{1}{\Gamma(1+\check{\alpha})} \int_{0}^{1} \vartheta^{\check{\alpha}}(1-\vartheta)^{\check{\alpha}}(2-\vartheta)^{m \check{\alpha}}\left|\mathcal{G}^{(3 \check{\alpha})}\left(\frac{1+\vartheta}{2} \eta_{1}+\frac{1-\vartheta}{2} \eta_{2}\right)\right|^{\rho}(d \vartheta)^{\check{\alpha}}\right)^{1 / \rho}\right] .
\end{aligned}
$$

Since $\left|\mathcal{G}^{(3 \check{)})}\right|^{\rho}$ is a generalized convex function on $\Omega$, we have

$$
\left|\mathcal{G}^{(3 \check{\alpha})}\left(\frac{1+\vartheta}{2} \eta_{1}+\frac{1-\vartheta}{2} \eta_{2}\right)\right|^{\rho} \leq\left(\frac{1+\vartheta}{2}\right)^{\check{\alpha}}\left|\mathcal{G}^{(3 \check{\alpha})}\left(\eta_{1}\right)\right|^{\rho}+\left(\frac{1-\vartheta}{2}\right)^{\check{\alpha}}\left|\mathcal{G}^{(3 \check{\alpha})}\left(\eta_{2}\right)\right|^{\rho}
$$

and

$$
\left|\mathcal{G}^{(3 \check{\alpha})}\left(\frac{1-\vartheta}{2} \eta_{1}+\frac{1+\vartheta}{2} \eta_{2}\right)\right|^{\rho} \leq\left(\frac{1-\vartheta}{2}\right)^{\check{\alpha}}\left|\mathcal{G}^{(3 \check{\alpha})}\left(\eta_{1}\right)\right|^{\rho}+\left(\frac{1+\vartheta}{2}\right)^{\check{\alpha}}\left|\mathcal{G}^{(3 \check{\alpha})}\left(\eta_{2}\right)\right|^{\rho} .
$$


It follows that

$$
\begin{aligned}
\mid \Gamma^{2}(1 & +\check{\alpha}) \mathcal{G}\left(\frac{\eta_{1}+\eta_{2}}{2}\right)+\left(\frac{\eta_{2}-\eta_{1}}{24}\right)^{\check{\alpha}} \Gamma(1+\check{\alpha})\left[\mathcal{G}^{(\check{\alpha})}\left(\eta_{2}\right)-\mathcal{G}^{(\check{\alpha})}\left(\eta_{1}\right)\right] \\
& -\left(\frac{1}{\eta_{2}-\eta_{1}}\right)^{\check{\alpha}} \Gamma^{3}(1+\check{\alpha})_{\eta_{1}} \mathcal{I}_{\eta_{2}}^{(\check{\alpha})} \mid \\
\leq & \left(\frac{\left(\eta_{2}-\eta_{1}\right)^{3}}{96}\right)^{\check{\alpha}}\left[\left(\frac{1}{\Gamma(1+\check{\alpha})} \int_{0}^{1} \vartheta^{\check{\alpha}}(1-\vartheta)^{\check{\alpha}}(2-\vartheta)^{\check{\alpha}\left(\frac{\rho-m}{\rho-1}\right)}(d \vartheta)^{\check{\alpha}}\right)^{1-1 / \rho}\right. \\
& \times\left(\frac{1}{\Gamma(1+\check{\alpha})} \int_{0}^{1} \vartheta^{\check{\alpha}}(1-\vartheta)^{\check{\alpha}}(2-\vartheta)^{m \check{\alpha}}\right. \\
& \left.\times\left[\left(\frac{1+\vartheta}{2}\right)^{\check{\alpha}}\left|\mathcal{G}^{(3 \check{\alpha})}\left(\eta_{1}\right)\right|^{\rho}+\left(\frac{1-\vartheta}{2}\right)^{\check{\alpha}}\left|\mathcal{G}^{(3 \check{\alpha})}\left(\eta_{2}\right)\right|^{\rho}\right](d \vartheta)^{\check{\alpha}}\right)^{1 / \rho} \\
& +\left(\frac{1}{\Gamma(1+\check{\alpha})} \int_{0}^{1} \vartheta^{\check{\alpha}}(1-\vartheta)^{\check{\alpha}}(2-\vartheta)^{\check{\alpha}\left(\frac{\rho-m}{\rho-1}\right)}(d \vartheta)^{\check{\alpha}}\right)^{1-1 / \rho} \\
& \times\left(\frac{1}{\Gamma(1+\check{\alpha})} \int_{0}^{1} \vartheta^{\check{\alpha}}(1-\vartheta)^{\check{\alpha}}(2-\vartheta)^{m \check{\alpha}}\right. \\
& \left.\left.\times\left[\left(\frac{1-\vartheta}{2}\right)^{\check{\alpha}}\left|\mathcal{G}^{(\check{3})}\left(\eta_{1}\right)\right|^{\rho}+\left(\frac{1+\vartheta}{2}\right)^{\check{\alpha}}\left|\mathcal{G}^{(3 \check{\alpha})}\left(\eta_{2}\right)\right|^{\rho}\right](d \vartheta)^{\check{\alpha}}\right)^{1 / \rho}\right] \\
\leq & \left(\frac{\left(\eta_{2}-\eta_{1}\right)^{3}}{96}\right)^{\check{\alpha}}\left(\Psi_{1}^{* * *}\right)^{1-1 / \rho}\left[\left[\left(\Psi_{2}^{* * *}\right)\left|\mathcal{G}^{(3 \check{\alpha})}\left(\eta_{1}\right)\right|^{\rho}+\left(\Psi_{3}^{* * *}\right)\left|\mathcal{G}^{(3 \check{\alpha})}\left(\eta_{2}\right)\right|^{\rho}\right]^{1 / \rho}\right. \\
& \left.+\left[\left(\Psi_{3}^{* * *}\right)\left|\mathcal{G}^{(3 \check{\alpha})}\left(\eta_{1}\right)\right|^{\rho}+\left(\Psi_{2}^{* * *}\right)\left|\mathcal{G}^{(\check{3} \check{\alpha})}\left(\eta_{2}\right)\right|^{\rho}\right]^{1 / \rho}\right],
\end{aligned}
$$

where we have used the equalities

$$
\begin{aligned}
\Psi_{1}^{* * *}= & \frac{1}{\Gamma(1+\check{\alpha})} \int_{0}^{1} \vartheta^{\check{\alpha}}(1-\vartheta)^{\check{\alpha}}(2-\vartheta)^{\check{\alpha}}\left(\frac{\rho-m}{\rho-1}\right)(d \vartheta)^{\check{\alpha}} \\
= & {\left[\frac{\Gamma(1+\check{\alpha})}{\Gamma(1+2 \check{\alpha})}-\frac{\Gamma(1+2 \check{\alpha})}{\Gamma(1+3 \check{\alpha})}\right]+\frac{\Gamma\left(1+\check{\alpha}\left(\frac{2 \rho-m-1}{\rho-1}\right)\right)}{\Gamma\left(1+\check{\alpha}\left(\frac{3 \rho-m-2}{\rho-1}\right)\right)}-\frac{\Gamma\left(1+\check{\alpha}\left(\frac{3 \rho-m-2}{\rho-1}\right)\right)}{\Gamma\left(1+\check{\alpha}\left(\frac{4 \rho-m-3}{\rho-1}\right)\right)}, } \\
\Psi_{2}^{* * *}= & \left(\frac{1}{2}\right)^{\check{\alpha}} \frac{1}{\Gamma(1+\check{\alpha})} \int_{0}^{1} \vartheta^{\check{\alpha}}(1-\vartheta)^{\check{\alpha}}(1+\vartheta)^{\check{\alpha}}(2-\vartheta)^{m \check{\alpha}}(d \vartheta)^{\check{\alpha}} \\
= & {\left[\frac{\Gamma(1+\check{\alpha})}{\Gamma(1+2 \check{\alpha})}+\frac{\Gamma(1+(m+1) \check{\alpha})}{\Gamma(1+(m+2) \check{\alpha})}-\frac{\Gamma(1+2 \check{\alpha})}{\Gamma(1+3 \check{\alpha})}-\frac{\Gamma(1+(m+2) \check{\alpha})}{\Gamma(1+(m+3) \check{\alpha})}\right] } \\
& -\left(\frac{1}{2}\right)^{\check{\alpha}}\left[\frac{\Gamma(1+2 \check{\alpha})}{\Gamma(1+3 \check{\alpha})}-\frac{\Gamma(1+3 \check{\alpha})}{\Gamma(1+4 \check{\alpha})}+\frac{\Gamma(1+(m+2) \check{\alpha})}{\Gamma(1+(m+3) \check{\alpha})}-\frac{\Gamma(1+(m+3) \check{\alpha})}{\Gamma(1+(m+4) \check{\alpha})}\right], \\
\Psi_{3}^{* * *}= & \left(\frac{1}{2}\right)^{\check{\alpha}} \frac{1}{\Gamma(1+\check{\alpha})} \int_{0}^{1} \vartheta^{\check{\alpha}}(1-\vartheta)^{\check{\alpha}}(1-\vartheta)^{\check{\alpha}}(2-\vartheta)^{m \check{\alpha}}(d \vartheta)^{\check{\alpha}} \\
= & \left(\frac{1}{2}\right)^{\check{\alpha}}\left[\frac{\Gamma(1+2 \check{\alpha})}{\Gamma(1+3 \check{\alpha})}-\frac{\Gamma(1+3 \check{\alpha})}{\Gamma(1+4 \check{\alpha})}+\frac{\Gamma(1+(m+2) \check{\alpha})}{\Gamma(1+(m+3) \check{\alpha})}-\frac{\Gamma(1+(m+3) \check{\alpha})}{\Gamma(1+(m+4) \check{\alpha})}\right] .
\end{aligned}
$$

This completes the proof of Theorem 6.5.

We have some particular cases of Theorem 6.5. 
Corollary 6.6 Under the suppositions of Theorem 6.5, we have the following conclusions.

(1) Choosing $m=0$, we get

$$
\begin{aligned}
\mid \Gamma^{2}(1+\check{\alpha}) \mathcal{G}\left(\frac{\eta_{1}+\eta_{2}}{2}\right)+\left(\frac{\eta_{2}-\eta_{1}}{24}\right)^{\check{\alpha}} \Gamma(1+\check{\alpha})\left[\mathcal{G}^{(\check{\alpha})}\left(\eta_{2}\right)-\mathcal{G}^{(\check{\alpha})}\left(\eta_{1}\right)\right] \\
\quad-\left(\frac{1}{\eta_{2}-\eta_{1}}\right)^{\check{\alpha}} \Gamma^{3}(1+\check{\alpha})_{\eta_{1}} \mathcal{I}_{\eta_{2}}^{(\check{\alpha})} \mid \\
\leq\left(\frac{\left(\eta_{2}-\eta_{1}\right)^{3}}{96}\right)^{\check{\alpha}}\left(\nabla_{1}^{*}\right)^{1-1 / \rho}\left[\left[\left(\nabla_{2}^{*}\right)\left|\mathcal{G}^{(3 \check{\alpha})}\left(\eta_{1}\right)\right|^{\rho}+\left(\nabla_{3}^{*}\right)\left|\mathcal{G}^{(3 \check{\alpha})}\left(\eta_{2}\right)\right|^{\rho}\right]^{1 / \rho}\right. \\
\left.\quad+\left[\left(\nabla_{3}^{*}\right)\left|\mathcal{G}^{(3 \check{\alpha})}\left(\eta_{1}\right)\right|^{\rho}+\left(\nabla_{2}^{*}\right)\left|\mathcal{G}^{(3 \check{\alpha})}\left(\eta_{2}\right)\right|^{\rho}\right]^{1 / \rho}\right],
\end{aligned}
$$

where

$$
\begin{aligned}
& \nabla_{1}^{*}=\left[\frac{\Gamma(1+\check{\alpha})}{\Gamma(1+2 \check{\alpha})}-\frac{\Gamma(1+2 \check{\alpha})}{\Gamma(1+3 \check{\alpha})}\right]+\frac{\Gamma\left(1+\check{\alpha}\left(\frac{2 \rho-1}{\rho-1}\right)\right)}{\Gamma\left(1+\check{\alpha}\left(\frac{3 \rho-2}{\rho-1}\right)\right)}-\frac{\Gamma\left(1+\check{\alpha}\left(\frac{3 \rho-2}{\rho-1}\right)\right)}{\Gamma\left(1+\check{\alpha}\left(\frac{4 \rho-3}{\rho-1}\right)\right)}, \\
& \nabla_{2}^{*}=2^{\check{\alpha}} \frac{\Gamma(1+\check{\alpha})}{\Gamma(1+2 \check{\alpha})}-3^{\check{\alpha}} \frac{\Gamma(1+2 \check{\alpha})}{\Gamma(1+3 \check{\alpha})}+\frac{\Gamma(1+3 \check{\alpha})}{\Gamma(1+4 \check{\alpha})}, \\
& \nabla_{3}^{*}=\left[\frac{\Gamma(1+2 \check{\alpha})}{\Gamma(1+3 \check{\alpha})}-\frac{\Gamma(1+3 \check{\alpha})}{\Gamma(1+4 \check{\alpha})}\right] .
\end{aligned}
$$

(2) Choosing $m=\rho$, we obtain

$$
\begin{aligned}
\mid \Gamma^{2}(1+\check{\alpha}) \mathcal{G}\left(\frac{\eta_{1}+\eta_{2}}{2}\right)+\left(\frac{\eta_{2}-\eta_{1}}{24}\right)^{\check{\alpha}} \Gamma(1+\check{\alpha})\left[\mathcal{G}^{(\check{\alpha})}\left(\eta_{2}\right)-\mathcal{G}^{(\check{\alpha})}\left(\eta_{1}\right)\right] \\
\quad-\left(\frac{1}{\eta_{2}-\eta_{1}}\right)^{\check{\alpha}} \Gamma^{3}(1+\check{\alpha})_{\eta_{1}} \mathcal{I}_{\eta_{2}}^{(\check{\alpha})} \mid \\
\leq\left(\frac{\left(\eta_{2}-\eta_{1}\right)^{3}}{96}\right)^{\check{\alpha}}\left(\nabla_{1}^{* *}\right)^{1-1 / \rho}\left[\left[\left(\nabla_{2}^{* *}\right)\left|\mathcal{G}^{(3 \check{\alpha})}\left(\eta_{1}\right)\right|^{\rho}+\left(\nabla_{3}^{* *}\right)\left|\mathcal{G}^{(3 \check{\alpha})}\left(\eta_{2}\right)\right|^{\rho}\right]^{1 / \rho}\right. \\
\left.\quad+\left[\left(\nabla_{3}^{* *}\right)\left|\mathcal{G}^{(3 \check{\alpha})}\left(\eta_{1}\right)\right|^{\rho}+\left(\nabla_{2}^{* *}\right)\left|\mathcal{G}^{(3 \check{\alpha})}\left(\eta_{2}\right)\right|^{\rho}\right]^{1 / \rho}\right],
\end{aligned}
$$

where

$$
\begin{aligned}
\nabla_{1}^{* *}= & 2^{\check{\alpha}}\left[\frac{\Gamma(1+\check{\alpha})}{\Gamma(1+2 \check{\alpha})}-\frac{\Gamma(1+2 \check{\alpha})}{\Gamma(1+3 \check{\alpha})}\right], \\
\nabla_{2}^{* *}= & {\left[\frac{\Gamma(1+\check{\alpha})}{\Gamma(1+2 \check{\alpha})}+\frac{\Gamma(1+(\rho+1) \check{\alpha})}{\Gamma(1+(\rho+2) \check{\alpha})}-\frac{\Gamma(1+2 \check{\alpha})}{\Gamma(1+3 \check{\alpha})}-\frac{\Gamma(1+(\rho+2) \check{\alpha})}{\Gamma(1+(\rho+3) \check{\alpha})}\right] } \\
& -\left(\frac{1}{2}\right)^{\check{\alpha}}\left[\frac{\Gamma(1+2 \check{\alpha})}{\Gamma(1+3 \check{\alpha})}-\frac{\Gamma(1+3 \check{\alpha})}{\Gamma(1+4 \check{\alpha})}+\frac{\Gamma(1+(\rho+2) \check{\alpha})}{\Gamma(1+(\rho+3) \check{\alpha})}-\frac{\Gamma(1+(\rho+3) \check{\alpha})}{\Gamma(1+(\rho+4) \check{\alpha})}\right], \\
\nabla_{3}^{* *}= & \left(\frac{1}{2}\right)^{\check{\alpha}}\left[\frac{\Gamma(1+2 \check{\alpha})}{\Gamma(1+3 \check{\alpha})}-\frac{\Gamma(1+3 \check{\alpha})}{\Gamma(1+4 \check{\alpha})}+\frac{\Gamma(1+(\rho+2) \check{\alpha})}{\Gamma(1+(\rho+3) \check{\alpha})}-\frac{\Gamma(1+(\rho+3) \check{\alpha})}{\Gamma(1+(\rho+4) \check{\alpha})}\right] .
\end{aligned}
$$

Remark 6.7 Letting $\check{\alpha}=1$, Theorem 6.5 reduces to Theorem 3.3 of [26]. 


\section{Examples}

Example 7.1 Let $\alpha=\frac{1}{2}, \eta_{1}=1, \eta_{2}=3, y \in(0,4)$, and $\mathcal{G}(y)=y^{3 \alpha}$. Then all the assumptions of Theorem 4.1 are satisfied.

Clearly,

$$
\begin{aligned}
& \left|\frac{\left(y-\eta_{1}\right)^{\check{\alpha}} \mathcal{G}\left(\eta_{1}\right)+\left(\eta_{2}-y\right)^{\check{\alpha}} \mathcal{G}\left(\eta_{2}\right)}{\left(\eta_{2}-\eta_{1}\right)^{\check{\alpha}}}-\frac{\Gamma(1+\check{\alpha})}{\left(\eta_{2}-\eta_{1}\right)^{\check{\alpha}} \eta_{1}} \mathcal{I}_{\eta_{2}}^{(\check{\alpha})} \mathcal{G}(u)\right| \\
& \quad=\left|\frac{(y-1)^{1 / 2} 1^{3 / 2}+(3-y)^{1 / 2} 3^{3 / 2}}{\sqrt{2}}-\frac{\Gamma(3 / 2)}{\sqrt{2}}{ }_{\mathcal{I}_{3}} \mathcal{I}^{(1 / 2)} u^{3 / 2}\right| \\
& \quad=\left|\frac{1+\sqrt{54}}{\sqrt{2}}-\frac{\sqrt{\pi}}{2 \sqrt{2}}(3 \sqrt{\pi})\right| \\
& \quad \approx 2.5711 .
\end{aligned}
$$

On the other hand, from Theorem 4.1 we get

$$
\begin{aligned}
& \frac{\left(y-\eta_{1}\right)^{2 \check{\alpha}}}{\left(\eta_{2}-\eta_{1}\right)^{\check{\alpha}}}\left[\left[\frac{\Gamma(1+\check{\alpha})}{\Gamma(1+2 \check{\alpha})}-\frac{\Gamma(1+2 \check{\alpha})}{\Gamma(1+3 \check{\alpha})}\right]\left|\mathcal{G}^{(\check{\alpha})}(y)\right|+\frac{\Gamma(1+2 \check{\alpha})}{\Gamma(1+3 \check{\alpha})}\left|\mathcal{G}^{(\check{\alpha})}\left(\eta_{1}\right)\right|\right] \\
& \quad+\frac{\left(\eta_{2}-y\right)^{2 \check{\alpha}}}{\left(\eta_{2}-\eta_{1}\right)^{\check{\alpha}}}\left[\left[\frac{\Gamma(1+\check{\alpha})}{\Gamma(1+2 \check{\alpha})}-\frac{\Gamma(1+2 \check{\alpha})}{\Gamma(1+3 \check{\alpha})}\right]\left|\mathcal{G}^{(\check{\alpha})}(y)\right|+\frac{\Gamma(1+2 \check{\alpha})}{\Gamma(1+3 \check{\alpha})}\left|\mathcal{G}^{(\check{\alpha})}\left(\eta_{2}\right)\right|\right] \\
& =\frac{(y-1)}{\sqrt{2}}\left[\left[\frac{\Gamma(3 / 2)}{\Gamma(2)}-\frac{\Gamma(2)}{\Gamma(5 / 2)}\right] \frac{\Gamma(5 / 2)}{\Gamma(2)}|y|+\frac{\Gamma(2)}{\Gamma(5 / 2)} \frac{\Gamma(5 / 2)}{\Gamma(2)}\right] \\
& \quad+\frac{(3-y)}{\sqrt{2}}\left[\left[\frac{\Gamma(3 / 2)}{\Gamma(2)}-\frac{\Gamma(2)}{\Gamma(5 / 2)}\right]|y|+\frac{\Gamma(2)}{\Gamma(5 / 2)} \frac{\Gamma(5 / 2)}{\Gamma(2)} 2\right] \\
& \approx 3.1287 .
\end{aligned}
$$

It is nice to see that the implication

$$
2.5711<3.1287
$$

holds in (7.1) and (7.2).

Example 7.2 Let $\alpha=1, \eta_{1}=0, \eta_{2}=3, y \in(-1 / 2, \infty)$, and $\mathcal{G}(y)=\ln (2 y+1)$. Then all the assumptions of Theorem 5.4 are satisfied.

We clearly see that

$$
\begin{aligned}
& \left|\left[\frac{\mathcal{G}\left(\eta_{1}\right)+\mathcal{G}\left(\eta_{2}\right)}{2^{\check{\alpha}}}\right] \Gamma(1+\check{\alpha})-\left(\frac{1}{\eta_{2}-\eta_{1}}\right)^{\check{\alpha}} \Gamma^{2}(1+\check{\alpha})_{\eta_{1}} \mathcal{I}_{\eta_{2}}^{(\check{\alpha})} \mathcal{G}(u)\right| \\
& \quad=\frac{\ln 7}{2}-\frac{1}{3}{ }_{0} \mathcal{I}_{3}^{(1)} \ln (2 u+1) \mid \\
& \quad \approx 0.9684 .
\end{aligned}
$$


On the other hand, we have

$$
\begin{aligned}
\Psi_{1}^{(\check{\alpha})} & :=\frac{1}{2^{\check{\alpha}}}\left[\frac{1}{\Gamma(1+\check{\alpha})}-\frac{\Gamma(1+\check{\alpha})}{\Gamma(1+2 \check{\alpha})}-\frac{\Gamma(1+2 \check{\alpha})}{\Gamma(1+3 \check{\alpha})}+\frac{\Gamma(1+3 \check{\alpha})}{\Gamma(1+4 \check{\alpha})}\right] \\
& =\frac{1}{2}\left[1-\frac{1}{2}-\frac{1}{3}+\frac{1}{4}\right]=\frac{5}{24}, \\
\Psi_{2}^{(\check{\alpha})} & :=\frac{1}{2^{\check{\alpha}}}\left[\frac{1}{\Gamma(1+\check{\alpha})}+\frac{\Gamma(1+\check{\alpha})}{\Gamma(1+2 \check{\alpha})}-\frac{\Gamma(1+2 \check{\alpha})}{\Gamma(1+3 \check{\alpha})}-\frac{\Gamma(1+3 \check{\alpha})}{\Gamma(1+4 \check{\alpha})}\right] \\
& =\frac{1}{2}\left[1+\frac{1}{2}-\frac{1}{3}-\frac{1}{4}\right]=\frac{11}{24},
\end{aligned}
$$

and

$$
\begin{aligned}
& \frac{\left(\eta_{2}-\eta_{1}\right)^{2 \check{\alpha}}}{16^{\check{\alpha}}}\left(\frac{1}{\Gamma(1+\check{\alpha})}-\frac{\Gamma(1+2 \check{\alpha})}{\Gamma(1+3 \check{\alpha})}\right)^{1-\frac{1}{\rho}}\left[\left[\Psi_{1}^{(\check{\alpha})}\left|\mathcal{G}^{(2 \check{\alpha})}\left(\eta_{1}\right)\right|^{\rho}+\Psi_{2}^{(\check{\alpha})}\left|\mathcal{G}^{(2 \check{\alpha})}\left(\eta_{2}\right)\right|^{\rho}\right]^{1 / \rho}\right. \\
& \left.\quad+\left[\Psi_{2}^{(\check{\alpha})}\left|\mathcal{G}^{(2 \check{\alpha})}\left(\eta_{1}\right)\right|^{\rho}+\Psi_{1}^{(\check{\alpha})}\left|\mathcal{G}^{(2 \check{\alpha})}\left(\eta_{2}\right)\right|^{\rho}\right]^{1 / \rho}\right] \\
& =\frac{9}{16} \sqrt{\frac{2}{3}}\left\{\left[\frac{80}{24}+\frac{176}{57624}\right]^{1 / 2}+\left[\frac{176}{24}+\frac{80}{57624}\right]^{1 / 2}\right\} \approx 1.2439 .
\end{aligned}
$$

Note that $0.9684<1.2439$, which gives the desired result in Theorem 5.4.

\section{Conclusion}

In the paper, we investigated the local fractional differentiation and integration for the generalized convex functions. Under this approach, we have derived three identities related to many well-known inequalities in the literature. For generalized convex functions, we obtained several novel bounds for higher-order local differentiable functions in different forms, which lead to the bounds of several known results in $[23,26]$. With reference to the definition of generalized convex functions, there is much to explore in the area of fractal analysis and machine learning by introducing specific values of the fractal parameters. Our ideas and approach may lead to a lot of follow-up research.

\section{Acknowledgements}

The authors would like to express their sincere thanks to the editor and anonymous reviewers for their helpful comments and suggestions.

Funding

The work was supported by the Natural Science Foundation of China (Grants Nos. 61673169, 11971142, 11401192).

Availability of data and materials

Not applicable.

Competing interests

The authors declare that they have no competing interests.

\section{Authors' contributions}

All authors contributed equally to the writing of this paper. All authors read and approved the final manuscript.

\section{Author details}

'Department of Mathematics, College of Science, Princess Nourah bint Abdulrahman University, Riyadh, Saudi Arabia.

2 Department of Mathematics, Government College University, Faisalabad, Pakistan. ${ }^{3}$ Department of Mathematics, Lahore College for Women University, Lahore, Pakistan. ${ }^{4}$ Department of Mathematics, Faculty of Arts and Sciences, Çankaya University, Ankara, Turkey. ${ }^{5}$ Department of Mathematics, Huzhou University, Huzhou, China. ${ }^{6}$ Hunan Provincial Key Laboratory of Mathematical Modeling and Analysis in Engineering, Changsha University of Science \& Technology, Changsha, China. 


\section{Publisher's Note}

Springer Nature remains neutral with regard to jurisdictional claims in published maps and institutional affiliations.

Received: 24 June 2020 Accepted: 16 November 2020 Published online: 23 November 2020

\section{References}

1. Yang, X.J.: Advanced Local Fractional Calculus and Its Applications. World Science Publisher, New York (2012)

2. Yang, X.-J., Tenreiro Machado, J.A.: A new fractal nonlinear Burgers' equation arising in the acoustic signals propagation. Math. Methods Appl. Sci. 42(18), 7539-7544 (2019)

3. Liu, J.-G., Yang, X.-J., Feng, Y.-Y., Cui, F.: On the (N+1)-dimensional local fractional reduced differential transform method and its applications. Math. Methods Appl. Sci. 43(15), 8856-8866 (2020)

4. Kumari, S., Kumari, M., Chugh, R.: Generation of new fractals via SP orbit with s-convexity. Int. J. Eng. Technol. 9(3), 2491-2504 (2017)

5. Kwun, Y.C., Shahid, A.A., Nazeer, W., Abbas, M., Kang, S.M.: Fractal generation via CR iteration scheme with s-convexity. IEEE Access 7, 69986-69997 (2019)

6. Shen, J.-M., Rashid, S., Noor, M.A., Ashraf, R., Chu, Y.-M.: Certain novel estimates within fractional calculus theory on time scales. AlMS Math. 5(6), 6073-6086 (2020)

7. Wang, L., Dai, L.-Z., Bian, H.-B., Ma, Y.-F., Zhang, J.-R.: Concrete cracking prediction under combined prestress and strand corrosion. Struct. Infrastruct. Eng. Maint. Manag. Life-Cycle Des. Perform. 15(3), 285-295 (2019)

8. Dai, L.-Z., Bian, H.-B., Wang, L., Potier-Ferry, M., Zhang, J.-R.: Prestress loss diagnostics in pretensioned concrete structures with corrosive cracking. J. Struct. Eng. 146(3), Article ID 04020013 (2020)

9. Yang, J., Baleanu, D., Yang, X.J.: Analysis of fractal wave equations by local fractional Fourier series method. Adv. Math. Phys. 2013, Article ID 632309 (2013)

10. Leveinen, J.: Composite model with fractional flow dimensions for well test analysis in fractured rocks. J. Hydrol. 234(3-4), 116-141 (2000)

11. Holm, S., Näsholm, S.P.: A causal and fractional all-frequency wave equation for lossy media. J. Acoust. Soc. Am. 130(4), 2195-2201 (2011)

12. Atangana, A., Dumitru, B.: New fractional derivatives with nonlocal and non-singular kernel: theory and application to heat transfer model. Therm. Sci. 20(2), 763-769 (2016)

13. Atangana, A., Koca, I.: Chaos in a simple nonlinear system with Atangana-Baleanu derivatives with fractional order. Chaos Solitons Fractals 89, 447-754 (2016)

14. Qi, H.-X., Yussouf, M., Mehmood, S., Chu, Y.-M., Farid, G.: Fractional integral versions of Hermite-Hadamard type inequality for generalized exponentially convexity. AIMS Math. 5(6), 6030-6042 (2020)

15. Sarikaya, M.Z., Budak, H., Erden, S.: On new inequalities of Simpson's type for generalized convex functions. Korean J. Math. 27(2), 279-295 (2019)

16. Kilıçman, A., Saleh, W.: Notions of generalized s-convex functions on fractal sets. J. Inequal. Appl. 2015, Article ID 312 (2015)

17. Mo, H.-X.: Generalized Hermite-Hadamard type inequalities involving local fractional integrals. https://arxiv.org/ftp/arxiv/papers/1410/1410.1062.pdf

18. Chen, G.-S., Srivastava, H.M., Wang, P., Wei, W.: Some further generalizations of Hölder's inequality and related results on fractal space. Abstr. Appl. Anal. 2014, Article ID 832802 (2014)

19. Sun, W.-B.: On generalization of some inequalities for generalized harmonically convex functions via local fractional integrals. Quaest. Math. 42(9), 1159-1183 (2019)

20. Shen, J.-M., Yang, Z.-H., Qian, W.-M., Zhang, W., Chu, Y.-M.: Sharp rational bounds for the gamma function. Math. Inequal. Appl. 23(3), 843-853 (2020)

21. Wang, M.-K., Chu, Y.-M., Li, Y.-M., Zhang, W.: Asymptotic expansion and bounds for complete elliptic integrals. Math. Inequal. Appl. 23(3), 821-841 (2020)

22. Hai, G.-J., Zhao, T.-H.: Monotonicity properties and bounds involving the two-parameter generalized Grötzsch ring function. J. Inequal. Appl. 2020, Article ID 66 (2020)

23. Kavurmaci, H., Avci, M., Özdemir, M.E.: New inequalities of Hermite-Hadamard type for convex functions with applications. J. Inequal. Appl. 2011, Article ID 86 (2011)

24. Dragomir, S.S., Agarwal, R.P.: Two inequalities for differentiable mappings and applications to special means of real numbers and to trapezoidal formula. Appl. Math. Lett. 11(5), 91-95 (1998)

25. Barani, A., Barani, S., Dragomir, S.S.: Refinements of Hermite-Hadamard inequalities for functions when a power of the absolute value of the second derivative is P-convex. J. Appl. Math. 2012, Article ID 615737 (2012)

26. Chun, L., Qi, F.: Integral inequalities of Hermite-Hadamard type for functions whose third derivatives are convex. J. Inequal. Appl. 2013, Article ID 451 (2013) 